\title{
Four reference models for transparency requirements in information systems
}

\author{
Mahmood Hosseini $^{1}$ (1) $\cdot$ Alimohammad Shahri $^{1} \cdot$ Keith Phalp $^{1} \cdot$ Raian Ali $^{1}$
}

Received: 22 June 2016/Accepted: 6 March 2017/Published online: 10 March 2017

(c) The Author(s) 2017. This article is published with open access at Springerlink.com

\begin{abstract}
Transparency is a key emerging requirement in modern businesses and their information systems. Transparency refers to the information which flows amongst stakeholders for the purpose of informed decision-making and taking the right action. Transparency is generally associated with positive connotations such as trust and accountability. However, it has been shown that it could have adverse effects such as information overload and affecting decisions objectiveness. This calls for systematic approaches for transparency to ensure its cost-effectiveness and avoid such adverse side effects. This is especially true considering that the relatively few works in the literature on transparency requirements have focused mainly on making information available and accessible and have paid little focus on the information receivers' side and making it meaningful for them. In this paper, we reflect on our previous research on transparency and its multi-faceted constructs and review multi-disciplinary conceptualisation and propose four reference models which are meant to form a holistic conceptual baseline for transparency requirements in information systems. These reference models cover transparency actors, transparency meaningfulness, transparency usefulness, and information quality in transparency. We also discuss the interdependencies amongst
\end{abstract}

Mahmood Hosseini

mhosseini@bournemouth.ac.uk

Alimohammad Shahri

ashahri@bournemouth.ac.uk

Keith Phalp

kphalp@bournemouth.ac.uk

Raian Ali

rali@bournemouth.ac.uk

1 Bournemouth University, Poole, UK these four reference models and their implications for requirements engineers and information system analysts. As a proof of concept, we analyse a mainstream transparency document, the United Kingdom Freedom of Information Act, in the light of our reference models and demonstrate the need to consider transparency more holistically and the need to include the information receiver's perspective and the inter-relations amongst various properties and constituents of transparency as well. We then highlight areas of improvement informed by our analysis.

Keywords Transparency requirements - Transparency stakeholders · Meaningful transparency $\cdot$ Useful transparency $\cdot$ Information quality $\cdot$ Transparency management $\cdot$ Information systems

\section{Introduction}

Transparency is derived from the Medieval Latin word "transparentum" and means showing light through [77]. Therefore, it is figuratively used to mean "being easily seen through". In its current usage, however, transparency is sometimes defined as "the open flow of information" [30] and "the release of information by institutions that is relevant to evaluating these institutions" [20]. Consequently, transparency is considered to be a requirement of businesses and their information systems, as it allows for making them accountable to their stakeholders and measuring their trustworthiness through their disclosure of relevant institutional information [61].

The positive connotation normally associated with transparency implies that it is a desirable quality and that it is always desirable for people and institutions to be 
transparent [50]. However, transparency might have adverse effects in certain cases as well. For example, it has been indicated that if precautionary steps are not taken towards providing transparency, transparency efforts may have a negative effect if clinicians avoid discussion because of the fear of feeling exposed or further upsetting patients and their families [39]. Consequently, precautionary steps in transparency provision should exist so that such adverse effects are minimised.

Transparency has been the subject of study in various domains, and there is a rich body of literature supporting it. In the domain of requirements engineering, however, transparency is currently under-researched and there has been a lack of conceptual models and rigorous methods which can be utilised for managing transparency. Furthermore, transparency is sometimes researched along with other information-related concepts, such as trust and privacy. Therefore, peculiarities and subtleties inherent to transparency, such as information overload and interpretability, are rarely paid scholarly attention to.

Moreover, transparency can be viewed as a regulatory or voluntary requirement. Regulatory requirements are generally about the compliance between system requirements and regulatory constraints. Such constraints could be enforced by law [24, 37] or they could be quality constraints enshrined perhaps by some form of contract or commitment [54]. Transparency might be seen as a regulatory requirement because laws and regulations may require organisations to be transparent for certain reasons and on certain processes [82]. Transparency can also be seen as a quality constraint, mainly as complying with information availability to the stakeholders who would need them [15]. Transparency could be even twinned with privacy and data protection in the sense of being transparent about the regulations about the right to hide or the obligation to reveal information [29].

Despite the existence of such established conceptualisations and requirements engineering approaches, little focus has been paid to transparency as an information receiver's requirement in information systems. In other words, information receivers have a wide range of metarequirements on the basic transparency requirements of making information available and accessible. This becomes more important when organisations decide to be transparent on a voluntary basis without the existence of constraints or regulatory requirements. In such cases, the main focus would be making transparency more meaningful and useful to the audience, the characteristic which has not been the main focus of various reviewed works in the requirements engineering literature on information systems. Our work provides a breadth of concepts attempting to pave the way to such a consideration.
A reference model is an abstract framework for understanding important relationships amongst the entities of a certain phenomenon, property, or system [46]. It facilitates the development of a specific reference using consistent standards or specifications supporting that phenomenon, property, or system. A reference model comprises a minimal set of unifying concepts, axioms, and relationships within a particular problem domain. It is independent of specific standards, technologies, or other concrete implementations details, but it intends to provide a common semantics that can be used unequivocally across and between different implementations [46].

The existence of reference models for a particular concept provides several benefits. First, reference models can facilitate discussion and evaluation and offer a comprehensive outlook on the problem domain. Second, reference models limit the scope of the study on that specific concept by concentrating on particular variables and defining the particular viewpoints which will help analysts and researchers in dealing with that concept. Third, they can be used as a foundation for the design and implementation of that concept. These benefits motivated us to develop a reference model for transparency as a distinct concept highlighting its peculiarities and uniqueness.

In this paper, we propose four reference models for transparency requirements in information systems in order to enable requirements engineers and information system analysts to better manage stakeholders' transparency requirements. These reference models capture: (1) the $a c$ tors involved in the process of transparency provision and the information flow amongst them, (2) the meaningfulness of the information made transparent through the disclosure of information, (3) the usefulness of such information for a particular audience in terms of providing them with decision-making capabilities through the disclosed information, and (4) the quality of the information disclosed to its stakeholders. Our reference models provide a foundation to measure and manage transparency as a first-class requirements engineering concept. These four reference models are built on top of the initial facets of transparency proposed in our position paper [32], which are in turn based on our extensive literature study on transparency in multiple disciplines including philosophy, management studies, business administration, journalism, and economy. The goal of our research in transparency, which stems from global trends such as open data movement, is to make quality information available in a meaningful and useful style to the right audience. As a proof of concept, we use our reference models to investigate the United Kingdom (UK) Freedom of Information Act and propose enhancements to it from the perspective of information receivers and transparency seekers. 
The rest of the paper is structured as follows. Section 2 describes the previous work on transparency in the domain of requirements engineering. Section 3 discusses our reference models. The first reference model, Transparency Actors Wheel, focuses on information circulation amongst relevant stakeholders. The second reference model, Transparency Depth Pyramid, centres on what constitutes meaningful transparency. The third reference model, Transparency Achievement Spectrum, concentrates on steps which should be taken in order to reach useful transparency. The fourth reference model, Information Quality in Transparency, is borrowed from the work of Kahn et al. [40] and identifies information quality dimensions. Section 4 covers interdependencies that exist amongst these four reference models and their implications for managing transparency requirements. Section 5 investigates the UK Freedom of Information Act with regard to the four reference models and provides a detailed discussion and possible amendments on it. Section 6 concludes this paper and presents our future work.

\section{Background and related work}

Transparency is generally defined as the open flow of information amongst stakeholders [30]. Because transparency deals with information, it becomes one of the main attributes of an information system. An information system is defined as a system which is designed to collect, organise, store, and communicate information to its stakeholders [43]. It is the academic study of networks of hardware and software that people and institutions use to accumulate, filter, manage, generate, and disseminate information [38].

From a requirements engineering perspective, the definition of transparency, based on the above definition, can be formatted as a user story as follows:

As stakeholder A, I want to get information from stakeholder B, so that I can use the information in my decision making.

Or as follows:

As stakeholder A, I want to give information to stakeholder B, so that stakeholder B can use the information in their decision making.

For example, an insurance company customer may need to get some information from the insurance company about their cancellation policies, so that they can decide whether they want to take that insurance product from that company or not. This is an example of transparency for the first user story. For another example, a bank provides information on different current account products and their comparison with each other to the bank customer, so that the bank customer can make an informed decision on what current account product to choose. This is an example of transparency for the second user story.

It can be argued that when one considers transparency as a main focus, they can manage that information flow better. For example, a piece of information could pass the security and privacy constraints, but it may still not provide transparency if it not understandable or actionable by the stakeholders. Catering for properties specific for transparency, such as understandability and information overload, would allow making that information flow economic and useful.

In the field of governance and politics, information transparency is regarded as a requirement of every citizen [3]. In the domain of software engineering, a software system is transparent when it makes the information it deals with transparent, along with its internal functioning process, which are called information transparency and process transparency respectively $[16,17]$, and when all its functionalities are disclosed to its users [49].

This is more technically illustrated in requirements engineering, where transparency is generally viewed as a non-functional requirement (NFR) of the stakeholders of a software system. Transparency is considered to be an NFR because it is orthogonal to software functionality since it can be viewed as a quality issue, and because software can work with or without it [17]. It is also advocated that transparency must be dealt with in the context of requirements specification [17].

However, the literature on transparency as an NFR is scarce. In one of the first works on transparency as an NFR [8], it is argued that transparency requirements can be managed using the NFR Framework [9] and $i^{*}$ modelling [83]. The authors also conclude that $i^{*}$ modelling is not the final answer to transparency, and certain augmentations might be needed for managing transparency requirements more efficiently with $i^{*}$ modelling.

Furthermore, a transparency ladder is proposed with the aim of helping organisations to distinguish what transparency is and how they can demonstrate transparency [17]. The transparency ladder contains the following five non-functional requirements of accessibility, usability, informativeness, understandability, and auditability, which must be achieved in order to reach transparency. However, it can be argued that the steps in the transparency ladder refer more to separate information attributes that must be fulfilled, rather than steps to achieving transparency, as will be discussed in the next section. Using the NFR Framework, a software transparency softgoal interdependency graph (SIG) is also proposed [17].

In two other works, transparency is shown to facilitate the elicitation of users' needs and requirements [13, 14]. 
Github is provided as an example of a transparent environment, in which users' transparent interactions result in revealing more users' requirements. Furthermore, it has been proposed that Argumentation Framework can be utilised to elicit transparency requirements of stakeholders [63]. However, there has been no work on reference models on transparency within the area of systems analysis and design in general and requirements engineering in particular. The existing models, e.g., the transparency ladder, are partial and are used for a different purpose.

The authors of this paper have also conducted several research works related to transparency in the field of requirements engineering. We proposed an initial model of transparency stakeholders and the information flow amongst them [31]. We also proposed several facets of transparency and categorised them into four groups of transparency stakeholders, transparency meaningfulness, transparency usefulness, and information quality [32]. Based on the facets of transparency, we also devised a modelling language, called TranspLan, which helps requirements engineers and information system analysts in eliciting, modelling, and analysing transparency requirements of stakeholders in business information systems [33]. This paper constructs and proposes four reference models for transparency requirements based on our previous results.

\section{Transparency reference models}

In this section, we will introduce our proposed reference models for managing transparency requirements in information systems. These reference models provide a foundation to measure and manage transparency as a first-class concept in information systems and requirements engineering. These four reference models are based on an extensive literature study on transparency in multiple disciplines including philosophy, management studies, business administration, journalism, and economy. The goal is to provide a solid foundation in the management of transparency requirements to make quality information available in a meaningful and useful style to the right audience.

\subsection{Reference model 1: Transparency Actors Wheel}

In order to understand transparency requirements, one essential prerequisite is to identify the relevant actors in an information exchange. Amongst other things, the identification of these actors makes it possible to understand where the information originates, which actors provide the information, which actors receive it, and whether certain channels are used to relay information.
An initial model of information exchange illustrating relevant actors exists, and in this model, which is presented in Fig. 1 (left-hand side), two entities are introduced, information source and information receiver [69]. The source disseminates some information to the receiver, and the receiver provides feedback based on that information back to the source. Based on this initial model, a more complex model of information exchange is proposed in order to fit today's social networks [69]. In this newer model of information exchange, presented in Fig. 1 (right-hand side), receivers can be a group of separate individuals instead of one person. Furthermore, a new entity called observer can observe the exchanged information and can have access to that. The observer may also engage in these exchanges.

While these models have satisfied the needs of those proposing them, some key elements are missing that are essential for the study of transparency. The first one is the information medium which relays the information. The consideration of an information exchange medium as a technical actor is essential because it is where information can be stored, and is therefore prone to information leakage and unwanted transparency. The example of Ashley Madison website (an online dating service for married people or people in committed relationships) and the problems caused by its hacking is one of the many examples depicting the significance of information exchange media in any transparency model of information exchange. The second missing element is information entity, i.e., the entity whose information is being exchanged. More often than not, information providers provide information which involves other entities, e.g., another person or organisation. It is therefore essential to consider them in any transparency model of information exchange. Third, the nature of information has not been thoroughly investigated in these information exchange models. Not all the information in an information exchange model relates to transparency. This is another point to be considered in a transparency model of information exchange.

The above reasons have been considered in Transparency Actors Wheel, which was initially proposed by the authors [34] and later refined [31]. This reference model proposes four actors in any information exchange model suited for the analysis of transparency requirements and is illustrated in Fig. 2. These actors are information provider (IP), information receiver (IR), information medium (IM), and information entity (IE). The model further divides the information flow amongst the actors into transparency-related information (TRI) and transparency-unrelated information (TUI), thus distinguishing between them.

It should be noted that in our transparency model of information exchange, IP, IR, or IE do not necessarily refer to one stakeholder. Therefore, IP could be a person, a group of people, an organisation, a group of organisations, or any 
Fig. 1 Initial information exchange model (left); model fitted for social networks (right)

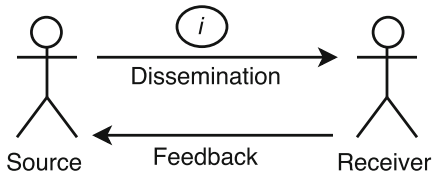

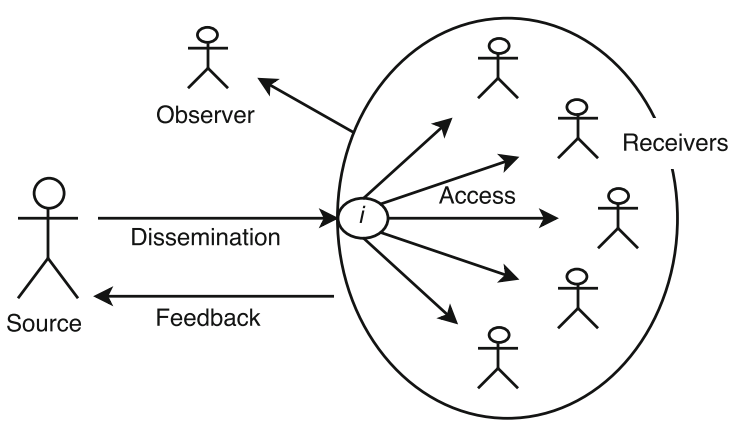

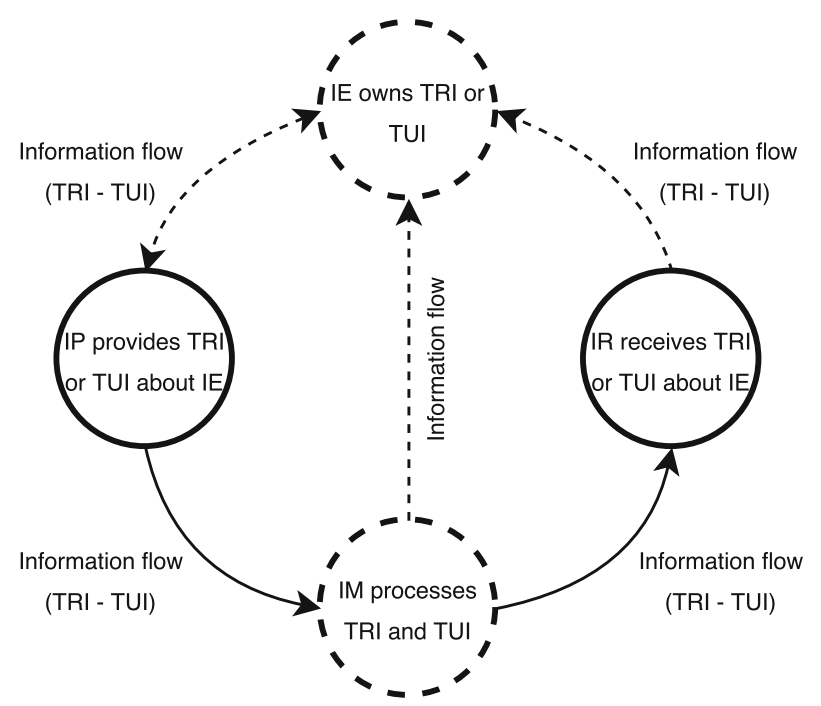

Legend

IP: Information Provider
IR: Information Receiver
IE: Information Entity
IM: Information Medium

TUI: Transparency-Unrelated Information TRI: Transparency-Related Information

Fig. 2 Transparency Actors Wheel (dotted lines show non-compulsory elements)

combination of those. The same rule applies to IM, i.e., IM can be one medium or a group of media fulfilling the role of information processing and relaying.

Transparency Actors Wheel is important for understanding the spread of transparency amongst the actors and for the detection of possible conflicts of interest amongst them [31]. Furthermore, it facilitates the classification of transparency based on its actors. Transparency can be classified into supply-side transparency and demand-side transparency [50]. In supply-side transparency, transparency is supplied by the information provider in two ways: it is either supplied voluntarily, as a means to increase information receivers' trust or increase information providers' accountability [45], or it is supplied coercively, as a means of complying with legal obligations. In demand-side transparency, transparency is provided in response to demands and public requests, by providing information which is otherwise inaccessible [21].

The legal obligations of information providers to supply transparency fall into three categories of mandatory transparency, discretionary transparency, and involuntary transparency [64]. Mandatory transparency refers to policies that oblige actors to disclose specific information, e.g.,
Freedom of Information Act. Discretionary transparency refers to policies that oblige actors to publish some information online, but do not specify what exactly should be disclosed, e.g., the website https://www.data.gov where federal agencies place online high-value datasets of their choice. Involuntary transparency refers to regulatory responses to whistleblowers and information leaks. This last type of supply-side transparency is also classified as non-agent-controlled transparency [44], where free independent third-party actors, such as the press, disclose information by wilfully investigating and reporting the activities of an agent. The word agent in this context clearly refers to the role of information provider. On the other hand, mandatory transparency and discretionary transparency are agent-controlled transparency, where information is disclosed by an agent in response to some requirements on the agent, such as Freedom of Information acts or personal demands, to make some information about its activities available.

We discuss that demand-side transparency can also fall into two categories of legal demands for transparency and personal demands for transparency. The first category, legal demands for transparency, denotes transparency 
requirements which are based on laws and regulations, such as Freedom of Information laws. It is important to recognise that such demand-side transparency creates a mandatory transparency on the supply-side as well. The second category, personal demands for transparency, denotes transparency requirements which are personal and as a result, place no obligations on the supply-side to provide transparency. Both categories of demand-side transparency are agent-controlled, because the agent (i.e., the information provider) has control over the amount of information which is disclosed.

Apart from the supply-side transparency and demandside transparency, we also advocate that medium-instilled transparency should also be considered. Frequently, the medium used to relay information between an information provider and an information receiver may lead to unwanted transparency as a result of information leakage. As such, this kind of transparency is categorised as non-agent-controlled, because the agent (i.e., the information provider) has no control over the volume of disclosed information. Fig. 3 summarises the discussions above.

\subsection{Reference model 2: Transparency Depth Pyramid (meaningful transparency)}

Transparency requirements can be divided into three main categories [4], which represent how meaningful the provided transparency is. These categories are primarily meant to deal with three questions and provide answers to them:

- Data transparency, or questions relating to data, content, and information: These questions primarily answer what information is needed and who are the stakeholders in the context of transparency. For example, in an online email service platform, data transparency reveals whether emails are secured using encryption techniques, or how big the attachments to an email can be.

- Process transparency, or questions relating to processes, behaviours, and interactions: These questions primarily answer how something is performed in the context of transparency. For example, in an online email service platform, process transparency reveals how emails are encrypted to become secure, or how attachments are scanned for malwares.

- Policy transparency, or questions relating to intentions, policies, and decision-making: These questions primarily answer why an action is performed in the context of transparency. For example, in an online email service platform, policy transparency reveals why the size of attachments are limited to a certain amount, or why an encryption technique is needed for securely delivering emails.

It has been pointed out that process transparency usually requires data transparency, and policy transparency usually requires data transparency and process transparency [4]. For example, disclosing why an encryption technique is required for the secure delivery of emails reveals the fact that the emails are encrypted and use an encrypted medium, and may also reveal some information about the encryption processes.

In another classification of transparency, transparency can be categorised as identity transparency, which makes transparent the identity of information exchangers, content transparency, which makes transparent the content and the changes to the content, and interaction transparency, which makes transparent the actions performed during the

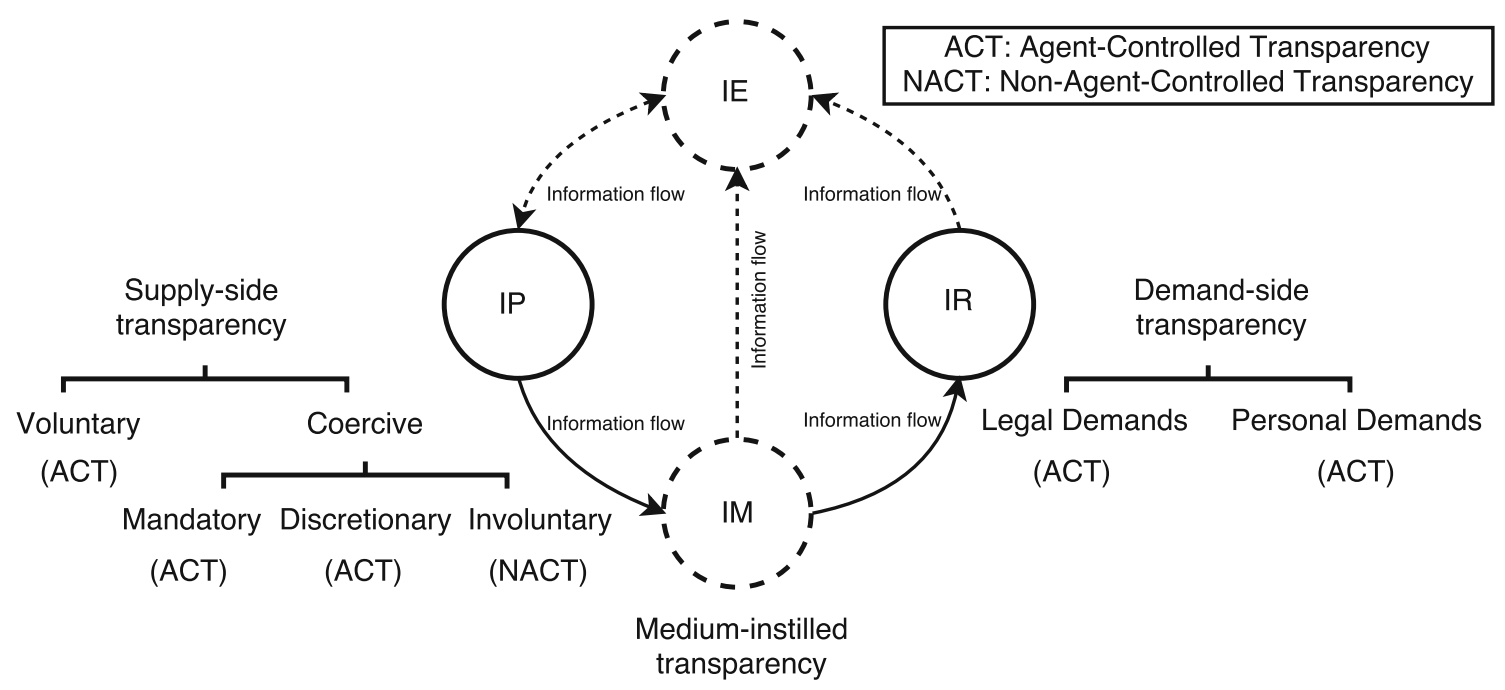

(NACT)

Fig. 3 Transparency Actors Wheel with transparency classifications 
interaction to a third-party observer [69]. This paper argues that the first two types of transparency, i.e., identity transparency and content transparency, fall into the category of data transparency, as identity and content comprise data, while interaction transparency falls into the category of process transparency, since interactions reveal a process of information exchange.

Proceeding from data transparency to process transparency and policy transparency gives depth to transparency, and the deeper transparency is provided, the more meaningful the information becomes to its stakeholders. Such a shift in transparency provision can lead to positive side effects, such as more trust. For example, it has been argued that citizens trust their governments more when they have access to and understand government decisionmaking processes [4].

Disclosing the 'why' will help build trust between information receivers and information providers. For example, stakeholders will trust a recommender system more and act upon its recommendations when it provides explanations why it has suggested a particular recommendation to them [65]. It will also prevent a practice known as window dressing, which is manipulating information by readjusting the composition of information. Revealing the reasoning makes it possible for stakeholders to spot possible flaws and also to identify whether the line of reasoning results in outcomes that match the disclosed data. The same argument applies for disclosing the 'how', but at a lower level, since the intentions of information providers remain hidden and only processes are disclosed.

Disclosing the 'how' will expose data cooking as well, which refers to the processing of raw data. Making the processes of providing information transparent to stakeholders means that stakeholders will know where the information is originated from, how it is represented, and how raw information is mediated before it reaches them. As highly mediated information provides greater chances for information misrepresentation and manipulation [50], it can potentially lead to a sub-optimal information flow [59], which can jeopardise transparency.

A systematic approach aiming for providing meaningful transparency during transparency management should therefore distinguish between data transparency, process transparency, and policy transparency. Furthermore, the management of transparency also needs to recognise and include other regulations and policies that can affect the disclosure of information. It needs to find the answers to the following questions in order to manage the meaningfulness of transparency.

- Does the disclosed information reveal processes and policies? How does such disclosure help stakeholders in their decision-making? Several studies define transparency as the extent to which one entity discloses relevant information about its own decision processes, procedures, performance, and functioning [12]. In order to provide process and policy transparency for the management of transparency requirements, the disclosed information should be analysed and categorised accordingly. The processes should be linked to data, should provide procedures upon request to avoid information overload, and should be presented clearly in a systematic way, e.g., chronologically. Policies should be linked to data (and processes), should provide reasons upon request to avoid information overload, and should be presented semantically, e.g., on a cause and effect basis. Feedback loops may be utilised to assist the management of transparency requirements in finding any discrepancies between data and processes/ policies and of outdated or emerging requirements.

- Does the disclosed information reveal stakeholders' identity information? What anonymity regulations exist that must be considered in managing transparency requirements? Concerning data transparency, it is also important to know whether it reveals any identity, self (i.e., personal), or hidden information, or that the data contains none of these elements. Revealing identity information can diminish, if not demolish, the anonymity of stakeholders where it is also a requirement of the stakeholders to remain anonymous. For example, in forums where people are expected to openly criticise an organisation's policies, transparency requirements must be governed by anonymity regulations.

- Does the disclosed information reveal stakeholders' self information? What privacy regulations exist that must be considered in managing transparency requirements? This is where transparency and privacy intersect and transparency may threaten stakeholders' privacy [48]. As revealing self information can endanger stakeholders' privacy requirements, it must be ensured at early stages of system analysis that the revealed data complies with privacy regulations by a systematic analysis of the disclosed data during the management of transparency requirements [7].

This should be noted that transparency is not the opposite of privacy, but there are occasions where the two concepts get at odds with each other, leading to conflicting demands between transparency and privacy [56]. Similarly, security and transparency are sometimes viewed as two antagonistic requirements which must be dealt with in the early phases of system analysis [7]. Therefore, transparency must be squared with values such as security and privacy [18]; otherwise, it can threaten both privacy and security, even though transparency is seen as a positive concept [48]. 


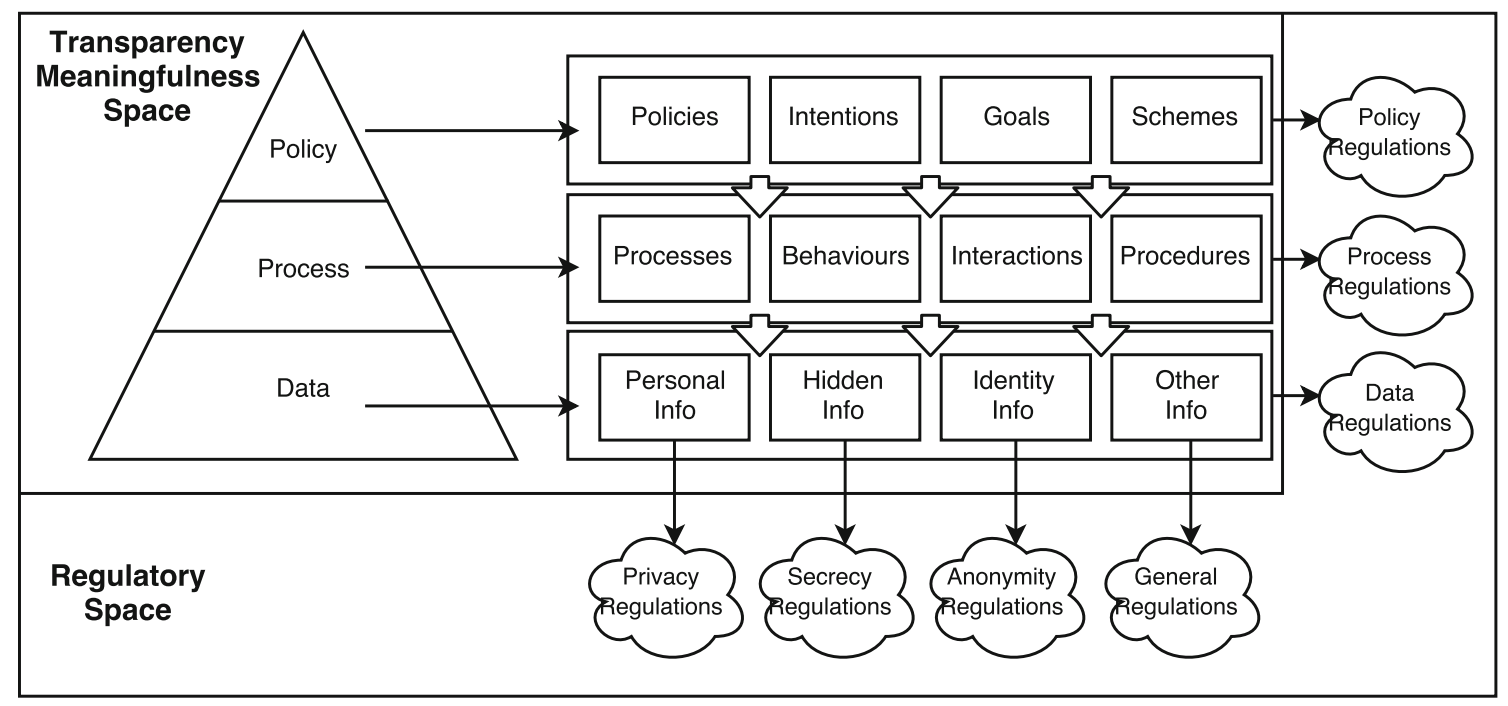

Fig. 4 Transparency Depth Pyramid (meaningful transparency)

Consequently, privacy and security are seen as two forces that can affect an organisations transparency [76].

- Does the disclosed information reveal stakeholders' hidden information? What secrecy regulations exist that must be considered in managing transparency requirements? Revealing the hidden information of stakeholders is in conflict with secrecy practices. Certain organisations, such as for-profit organisations, maintain a level of secrecy in order to have the market advantage over their competitors. However, it has been suggested that while organisations are justified to keep certain information secret, the justifications themselves should be made public and transparent [6]. During the management of transparency requirements, the secrecy policies of organisations must be reviewed as a measure against the disclosure of such information, while justifications for such secrecy regulations should be composed as part of the transparency management.

Figure 4 summarises the above discussion by proposing a Transparency Depth Pyramid, which implies a bottom-up structure for providing meaningful transparency from data transparency to process and policy transparency. It also illustrates the regulatory space which includes regulations for governing data, process, and policy transparency.

\subsection{Reference model 3: Transparency Achievement Spectrum (useful transparency)}

Useful transparency can be achieved when it enables stakeholders to make decisions based on the provided information and act upon them. For example, in the sociological and psychological sense, transparency is defined as gaining information and knowledge about the environment in order to prepare actions and decisions [22]. However, there are many steps between information availability and information actionability to consider. This section of the paper discusses the steps which should be taken towards achieving useful transparency.

\subsubsection{Information availability}

Information availability is the first step in achieving useful transparency. Obviously, no transparency is achieved if information providers withhold information from relevant information receivers. While making information available to information receivers, information providers should ensure that information quality is maintained to avoid problems such as wrong information, biased information, incomplete information, and information overload [42]. Correctness [51], completeness [26], and timeliness [27] are amongst these information qualities. It has also been noted that information disclosure alone may defeat the notion of transparency, because it can be obfuscating instead of enlightening [58]. Therefore, other steps are necessary to ensure a useful transparency is achieved.

\subsubsection{Information interpretation}

Information interpretation is the second step in achieving useful transparency. In several cases, the information provided by people, organisations, and government agencies are in forms and structures that are not comprehensible by information receivers. These forms can include cluttered tables, complicated charts, crowded figures, and lengthy texts. End-User Licence Agreements (EULAs) and privacy policies are two examples of such incomprehensible forms 
of information which need interpretation for the common reader. Therefore, it is usually essential for information providers, or mediators involved in transparency provision, such as journalists and reporters, to interpret the information in a way that can be easily understood by information receivers. As the President of European Parliament said: "There is no point in putting a report adopted in plenary online if no effort is made to explain it" [26].

Several studies highlight the importance of interpreting the provided information. For example, it is argued that a public service is called transparent when they inform stakeholders as well as explain their decisions to them [67]. Also, in their open learner model, the authors state that to achieve a useful kind of transparency, providing an interpretive mechanism is necessary to translate the information from a pedagogical perspective (i.e., the information provider's perspective) to a learner's perspective (i.e., the information receiver's perspective) in order to make the information comprehensible [73].

Since information interpretation can be affected by its mediators, it is essential that mediators present a truthful view of information to information receivers if transparency requirements are to be efficiently met. In any case, it has been observed that the number of mediators should be kept to a minimum, and it is better that information receivers access the information straight from the source rather than from mediators in an attempt to reduce information bias [78]. This, however, may affect information interpretability. Therefore, there is a need to find a trade off between the presence of mediators and their impact on the interpreted information.

Furthermore, given the probable diversity in information receivers' cognitive abilities, in order to manage transparency requirements one may actually have to find several different methods of information interpretation and representation, each of which suiting a different set of information receivers. These methods can then be used during requirements validation and further when the software system is being tested, to verify the success of information interpretation from information receivers' point of view. For example, in order to manage transparency requirements one may validate and test the use of charts and tables to present information systematically (e.g., similar to arrival and departure tables at airports), the use of different colours each with its own meaning (e.g., similar to those used in food industry on products labels), the use of a ranking or rating system to enhance comparison capabilities (e.g., similar to university rankings), and audiovisual aids to decrease reading and learning overhead (e.g., token displays with voice announcement).

\subsubsection{Information accessibility}

Information accessibility is the third step in achieving useful transparency. While information availability and interpretation are provided by information providers, information accessibility focuses on the ability of information receivers to access information. Sometimes referred to as information visibility [50], it is the degree to which information can be easily located by information receivers.

Several studies address information accessibility. For example, it is discussed that to achieve transparency, society members should have access to high-quality information [81]. Furthermore, it is maintained that transparency is not fully achieved unless the general public are aware of information availability and know how to access such information [5].

It should be noted that information availability per se does not guarantee its access [50]. Therefore, it must be ensured during the management of transparency requirements that the information is comfortably accessible by information receivers upon request. Furthermore, from the information receivers' point of view, inaccessible information and unavailable information cannot be distinguished from each other in several cases, because when they cannot access the information they may simply conclude that it is not initially available from information providers. For example, this is the case with lengthy terms and conditions and privacy policies, which usually make it difficult for their readers to locate and access the information they need. Therefore, it should be investigated as part of transparency management whether information availability requests are in reality difficulties in information accessibility.

\subsubsection{Information perception}

Information perception is the fourth step in achieving useful transparency. It refers to information receivers' perception of transparency once they have accessed the provided information. It acts at the cognitive level of information receivers and is therefore difficult to assess [72]. Furthermore, individual and psychological factors, such as confirmation bias, can influence the perceived level of transparency, as opposed to the actual level of transparency [78].

Several issues must be noted in dealing with information receivers' perception of information. If information receivers' perception of transparency does not match that of the information providers, useful transparency may fail to be achieved. Furthermore, if the provided information fails to change the already confirmed perception of an information receiver about the information provider, transparency may 
still not be achieved. Changing people's perception is not an easy task, and it needs continuous exposure to structured information which utilises their information processing methods [41] and it also requires constant social interactions with the people [71]. Since perception is subjective, different information receivers perceive the same information in different ways, and they respond to information according to their own perception [70].

As a possible solution, some perceptual obstacles may be overcome over time during the management of transparency requirements by putting importance on information receivers' feedback [1], which may also help in building trust relationships with them [52], which in turn may result in altered information receivers' perception of information providers. There is also a need for more studies by relevant communities to address the lack of metrics for evaluating information perception related to transparency.

\subsubsection{Information understandability}

Information understandability is the fifth step in achieving useful transparency. Obviously, information accessibility is a necessary condition for transparency, but insufficient on its own [50]. Therefore, for achieving useful transparency, information should also be understood and comprehended by information receivers. Therefore, understandability is sometimes considered as one of the two crucial dimensions of transparency [30].

Some studies have mentioned information understandability as one of the steps towards useful transparency. For example, it is pointed out that transparency can only be useful when it enhances understanding, not just increasing the flow of information [80]. Similarly, it has been argued that regulations on transparency must be enforced by governments to make available information more understandable to the public, because without such understanding, disclosed information will provide little de facto transparency [18].

From a transparency management perspective, the peculiarities discussed in information interpretation applies here as well, but the focus changes from information providers to information receivers. Furthermore, understanding is a complicated, personal experience [11], which does not necessarily relate to information interpretation. For example, while all students in a classroom receive the same information from a lecturer, their understanding of the subject (even technical subjects which leave little room for personal interpretations) may vary greatly. Therefore, the simplest representation of information should be chosen during transparency management. Alternatively, information receivers should be able to choose from various representations of information the one which maximises their understanding. Furthermore, culture, language, and cognitive abilities can impact understanding and learning [10] and consequently should be considered during transparency provision. Finally, a continuous feedback loop can be provided to information receivers during the management of transparency requirements in order to ensure the interpreted information intended by information providers matches, at least closely, the understood information by information receivers, and then software system adaptation can be planned accordingly.

\subsubsection{Information acceptance}

Information acceptance is the sixth step in achieving useful transparency. It implies either information receivers' perception of information matches their established beliefs and knowledge, in which case the new information confirms it, or that their perception of information does not match their established beliefs and knowledge, but the information changes it nonetheless. If information is not accepted by information receivers for any reason (personal or otherwise), then useful transparency may not be achieved.

While several studies consider information acceptance as an important step in achieving transparency [25, 80], there are no models or theories tailoring it systematically for transparency. However, several models and theories of individual acceptance, such as theory of planned behaviour, theory of reasoned action, and social cognitive theory already exist, which have been extended to suit other fields of study, such as information technology [79]. Similar research needs to be conducted in the management of transparency requirements.

Similar to information perception, information acceptance acts at the cognitive level of information receivers. Therefore, it is essential that different disciplines, such as psychology, be consulted and collaborated with in order to provide a holistic view of such cognitive aspects of transparency.

\subsubsection{Information actionability}

Information actionability is the seventh and last step in achieving useful transparency. Sometimes referred to as informed decision-making, information actionability emphasises that transparency becomes useful when the provided information enables information receivers to act upon it, make informed decisions, and therefore make use of the information. Information that does not change perceptions, does not help decision-making, or cannot be acted upon, does not constitute useful transparency.

Information actionability has been discussed in several studies. For example, it is argued that transparency is achieved when decision-makers receive the information 
essential to make sound decisions [47]. Similarly, it is argued that information availability and accessibility are not enough to reach transparency, and it is necessary for information receivers to do something they find important and valuable based on the provided information [28]. In the same fashion, the importance of information usability, i.e., using the obtained information by information receivers for performing an action or making a decision, is also emphasised [60].

Improper actions and partial or misled decisions are possible symptoms of where useful transparency has failed to be achieved. They can alert information providers to revise their transparency policies and transparency provision channels and techniques in an attempt to find loopholes and deficiencies. Furthermore, the management of transparency requirements can also benefit from reverse engineering on information receivers' actions and decisions based on the provided information in order to understand whether the information has served its purpose well, i.e., achieving useful transparency.

Figure 5 illustrates Transparency Achievement Spectrum, which illustrates the seven steps required in order to achieve useful transparency. For each step, an example of an influential factor is also provided in bubbles inside the reference model. It is worth emphasising that there are several other influential factors related to each step in providing useful transparency.

\subsubsection{Transparency usefulness and transparency meaningfulness}

It is essential for requirements engineers and information system analysts to recognise the difference between meaningful transparency and useful transparency. While meaningful transparency argues that information receivers must know the actions and reasons behind the provided information [26], useful transparency discusses that information provision should lead to information receivers' actionability and help in their decision-making processes, or at least to a change in their perception of the information provider [60]. Therefore, with regard to the disclosed information to information receivers, meaningful transparency can be considered as a static property of transparency, while useful transparency can be thought of as a dynamic property of transparency.

\subsection{Reference model 4: information quality in transparency}

Information quality in transparency is a crucial facet, as without it, transparency can hardly be reached. The literature on transparency does discuss the importance of information quality and provides some facets for it $[26,58]$. However, the literature on transparency is sometimes ambiguous how these information quality dimensions

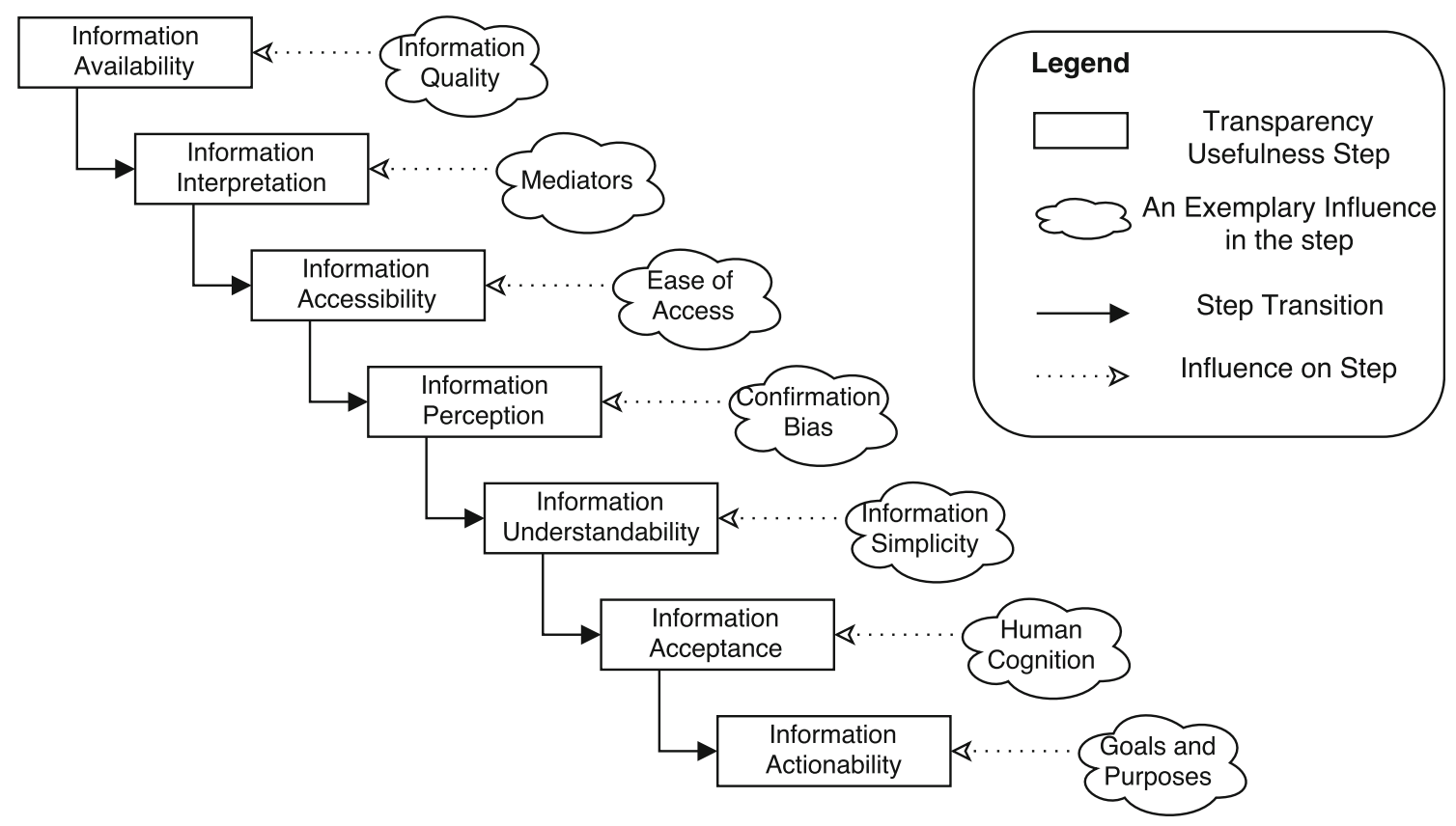

Fig. 5 Transparency Achievement Spectrum (useful transparency) 
should be fulfilled and by which stakeholders, and how their fulfilment can be assured. In the following, we briefly discuss four categories of information quality which can be used in transparency and the dimensions associated with them, borrowed from the work of Kahn et al. [40]:

- Sound information represents the quality of the information supplied by the information provider and consists of the following information quality dimensions: free of error, concise representation, completeness, and consistent representation.

- Dependable information represents the quality of the service in providing information by the information provider and consists of the following information quality dimensions: timeliness and security.

- Useful information represents the meeting/exceeding of the information receiver's expectations in the supplied information quality and consists of the following information quality dimensions: appropriate amount, relevancy, understandability, interpretability, and objectivity.

- Usable information represents the meeting/exceeding of the information receiver's expectations in information provision service and consists of the following information quality dimensions: believability, accessibility, ease of manipulation, reputation, and valueadded.

In the following, a brief definition for each of the information quality dimension is provided in an alphabetical order based on the work of Kahn et al. [40].

- Accessibility: The extent to which information is available, or easily and quickly retrievable.

- Appropriate amount: The extent to which the volume of information is suitable for the task at hand.

- Believability: The extent to which information is considered as true and credible.

- Completeness: The extent to which information is not missing and is of sufficient breadth and depth for the task at hand.

- Concise representation: The extent to which information is compactly represented.

- Consistent representation: The extent to which information is presented in the same layout.

- Ease of manipulation: The extent to which information is easy to manipulate and apply to different tasks.

- Free of error: The extent to which information is accurate and dependable.

- Interpretability: The extent to which information is in appropriate languages, symbols, and units, and the definitions are clear.

- Objectivity: The extent to which information is unbiased, unprejudiced, and impartial.

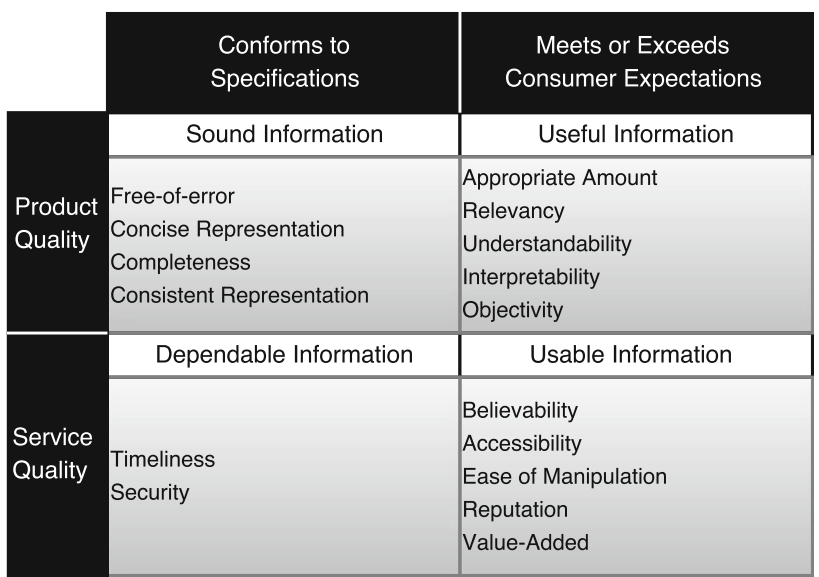

Fig. 6 Information quality dimensions [40]

- Relevancy: The extent to which information is applicable and helpful for the task at hand.

- Reputation: The extent to which information is highly regarded in terms of its source or content.

- Security: The extent to which access to information is restricted appropriately to maintain its security.

- Timeliness: The extent to which information is sufficiently up-to-date for the task at hand.

- Understandability: The extent to which information is easily comprehended.

- Value-added: The extent to which information is beneficial and provides advantages from its use.

It should be noted that interpretability and objectivity fall between some of these four categories, although they are categorised as useful information [40]. Objectivity can be categorised in either sound information or useful information, while interpretability can be classified in any of the four categories of sound information, useful information, dependable information, or usable information [40].

Figure 6 illustrates the information quality dimensions and their classifications.

\section{Interdependencies amongst models}

The four reference models for transparency provide a holistic view of transparency facets we need to consider during transparency provision. These reference models, however, have some interdependencies amongst each other as well. As there are four reference models, there will be six types of interdependencies in total, obtained by the following formula:

\section{Total Number of Dependencies Between $N$}

$$
\text { = } 4 \text { Models: } N *(N-1) / 2=4 *(4-1) / 2=6
$$

In this section, we will review and reflect upon all these interdependencies. We will use a running example in this 
section to communicate these interdependencies in a more comprehensible fashion. This running example involves a public relations office inside a financial institute who wants to disclose some information about the institute and their financial activities in the past year to the institute's customers and stakeholders through the institute website. In our example, the financial institute is the information entity, the public relations office is the information provider, customers and stakeholders are information receivers, and the institute website is the information medium.

\subsection{Interdependencies between information quality dimensions and Transparency Actors Wheel}

The information quality reference model clearly distinguishes between those quality dimensions which should conform to specifications, and those which should meet (or exceed) the expectations of the consumer. In this subsection, we will discuss how this distinction can be utilised in an effort to identify which stakeholders are involved in each category of information quality.

The first category of information quality relates to those about product quality which conform to specifications, such as having a concise or consistent representation. These quality dimensions can be fulfilled without the need to involve information receivers, though they might be able to help find problems and issues. Information providers can independently ensure the quality of these dimensions. As the provided information is about an information entity, they are also responsible to guarantee the quality of these dimensions. Information medium, similar to information receiver, is also not involved in this category.

In our running example, four information quality dimensions of being free of errors, completeness, concise representation, and consistent representation can be guaranteed by the financial institute and also by the public relations office. They can ensure all reports are correct, all figures have a concise and consistent representation and that the complete set of information is reported to the customers.

The second category of information quality relates to those about service quality which conform to specifications, namely security and timeliness. Similar to the first category, both information provider and information entity are involved in ensuring these quality dimensions. However, information medium also plays a role in this category, as it can affect both the timeliness and the security of the provided information. Guaranteeing these information quality dimensions does not involve information receiver, although they can be helpful in finding issues with these quality dimensions.

In our running example, public relations office may not be able to provide timely information if the financial institute does not provide them with the information in a timely manner. The institute website may also be down, affecting the timeliness of the provided information, or its security might have been compromised, affecting the security of the provided information.

The third category of information quality relates to those about product quality which meet or exceed consumer expectations, such as relevancy and interpretability. Information receivers are mainly engaged here, and only they can ensure whether qualities such as relevancy or understandability of information are achieved. However, two information qualities in this category, interpretability and objectivity, are affected by information provider and information entity as well. Therefore, these two transparency actors are also involved in guaranteeing these information quality dimensions. This is in line with propositions made in the information quality reference model about interpretability and objectivity being borderline dimensions [40]. Information medium, on the other hand, is not involved as it does not affect any of these information quality dimensions.

In our running example, public relations office may interpret the large quantity of data on spreadsheets and annual reports in a way that customers understand and make decisions based on it, while the customers decide whether the provided information has an appropriate amount, is relevant to their decision-making processes, and can be easily understood. As the financial institute creates the information, they can affect the objectivity and the interpretation of the provided information.

The fourth category of information quality relates to those about service quality which meet or exceed consumer expectations, such as believability and reputation. Similar to the previous category, information receivers are mainly involved in this category in deciding whether these information quality dimensions are properly met. However, one information quality dimension, accessibility, is also affected by information medium. Therefore, these two transparency actors should be linked to this fourth category.

Figure 7 illustrates different categories of information quality dimensions and transparency actors involved in each category.

\subsection{Interdependencies between information quality dimensions and transparency meaningfulness}

As information pieces are present in data, process, and policy, all information quality dimensions are linked to them. Arguably though, the link gets weaker for those quality dimensions which meet or exceed consumer expectations. For instance, there is no difference in checking completeness in data, process, or policy, and they all follow the same procedure, while for objectivity, one 


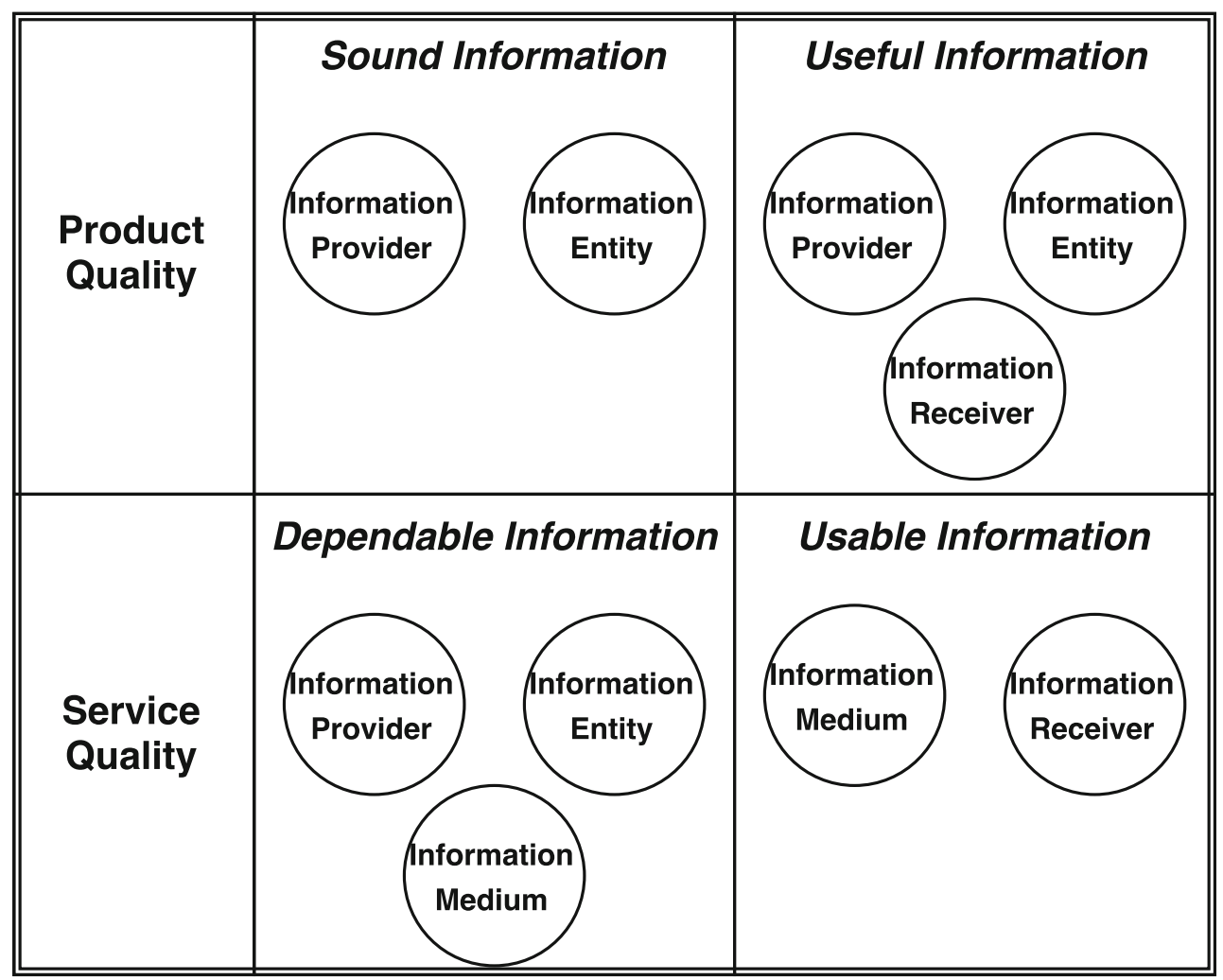

Fig. 7 Interdependencies between quality dimensions and transparency actors

may argue that it is easier to guarantee data objectivity than process or policy objectivity. For those information quality dimensions that meet or exceed consumer expectations, the link is strong with data transparency because it is relatively easy to check data quality, while it gets weak as it moves from data transparency to process transparency, and even weaker with the transition from process transparency to policy transparency, as it is harder to check information quality in processes than data and harder to check information quality in policies than processes.

In our running example, the financial institute may make available all the data, processes, and policies within their organisation. Checking for errors and problems in data documents follows the same standards and procedures as process and policy documents. So is the case for guaranteeing that all these documents have a concise and consistent representation, that they are complete, disclosed timely, and to the intended audience (i.e., the security perspective). For customers, on the other hand, it is easier to check whether the data is objective than to check whether the policy is objective, because data documents deal with facts while process and policy documents discuss procedures and goals of the institute which are less tangible to the customers. In the same fashion, documents containing data might be generally more accessible than documents containing processes and policies, might be easier to manipulate as they correspond to spreadsheets, fact sheets, charts, and graphs, and their added value can be more trusted and relied upon.

Figure 8 illustrates information quality dimensions and their links to transparency meaningfulness regarding the strength of the links.

\subsection{Interdependencies between information quality dimensions and transparency usefulness}

Each step in Transparency Achievement Spectrum, which denotes the level of transparency usefulness, can be mapped to one or more information quality dimensions, therefore highlighting the interdependencies between the Transparency Achievement Spectrum and information quality reference model.

Information availability, as the first step in achieving transparency usefulness, is linked to all information quality dimensions related to information entity and information provider, i.e., free of error, concise representation, completeness, consistent representation, timeliness, and security. This implies that the available information should already meet all the quality dimensions which are expected from information provider and information entity. Information interpretation clearly links to interpretability, while information accessibility has a clear link to accessibility. 


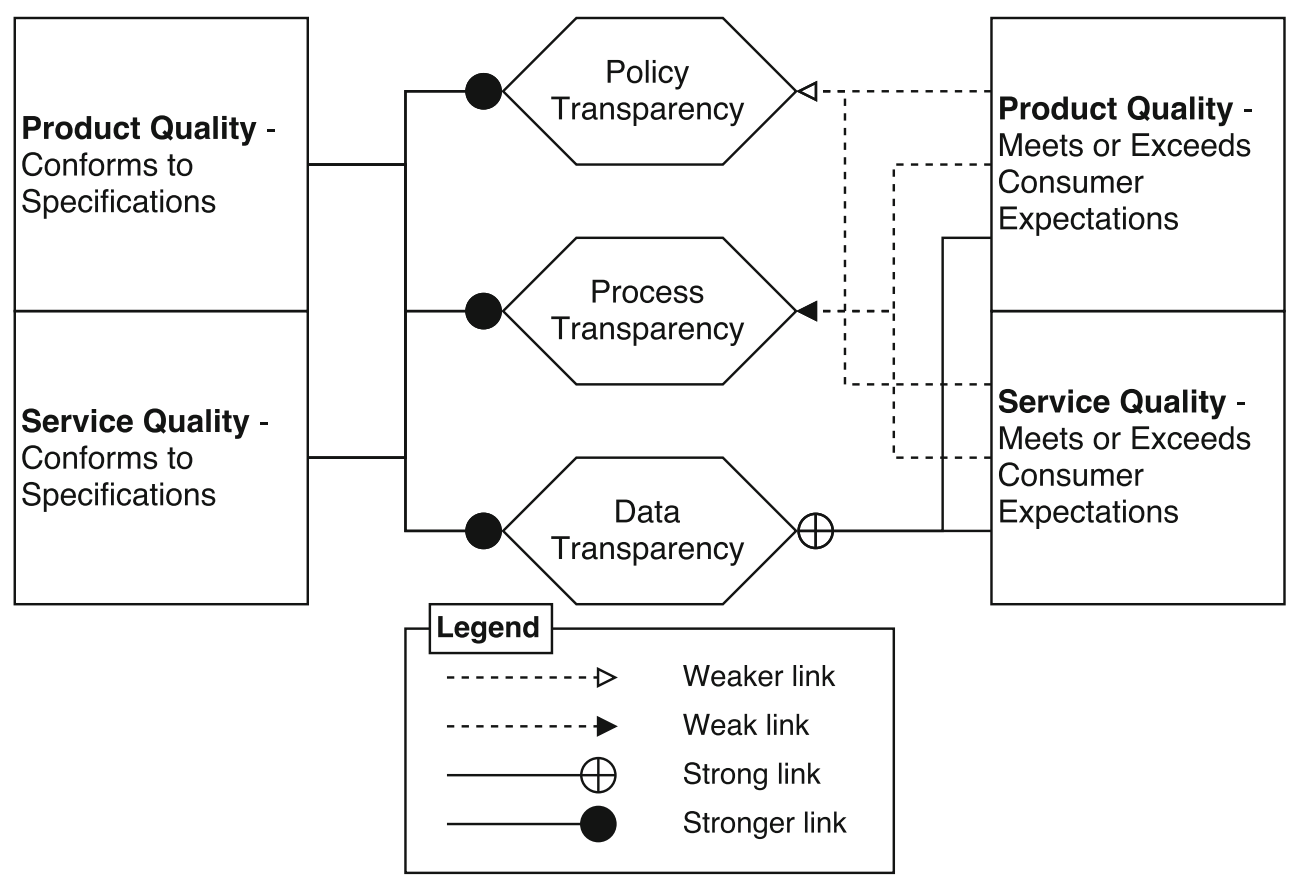

Fig. 8 Interdependencies between information quality dimensions and transparency meaningfulness

Information perception has a link to objectivity, as objective information, or its opposite, biased information, can have an influence on information receiver's perception of the provided information [57]. Reputation of the information provider or information entity also plays a key role in information receiver's perception [23], and is therefore linked to information perception.

Information understandability has a clear link to understandability, but is also linked to the appropriate amount of disclosed information, because studies show that too little or too much information can lead to information starvation and information overload, which in turn will affect the level of understandability in information receiver [74].

Information acceptance has a clear link to believability and is also linked to the reputation of the information provider or information entity, as their reputation is key to the acceptance of information by their information receivers. Information actionability is linked to relevance, as irrelevant information means the information has no role in information receiver's decision-making [68]. It is also linked to ease of manipulation, because ease of manipulation implies that information is easy to apply to different tasks by information receiver, which makes the information actionable. It is also linked to value-added characteristic, as added value implies that information is beneficial and provides advantages from its use, which again corresponds to actionable information.

Figure 9 illustrates information quality dimensions and the earliest step in transparency usefulness where they play their roles.

\subsection{Interdependencies between transparency usefulness and transparency actors}

Different transparency actors play their roles in different steps mentioned in Transparency Achievement Spectrum. Information entity is associated with information availability and information interpretation, as they are the owners or creators of information. They are also associated with information perception, as their reputation can help or harm information receiver's perception of the provided information. The same logic applies to information provider, as they are the source of information provision to information receivers.

Information medium is associated with information availability and accessibility. In our running example, availability is influenced by the institute website if the website is down or experiencing technical difficulties. But even when the information is available on the institute website, a bad design might hinder access to such information. Website design issues, such as poor search facilities or too many clicks before the information becomes accessible to information receivers, can harm the ease of access to information.

Information receiver is associated with information accessibility, because the final access to information can also be determined by information receiver's skills and capabilities. In our running example, the financial institute information on their website may simply be inaccessible by some stakeholders who do not possess the necessary knowledge to surf the internet, do not have the 

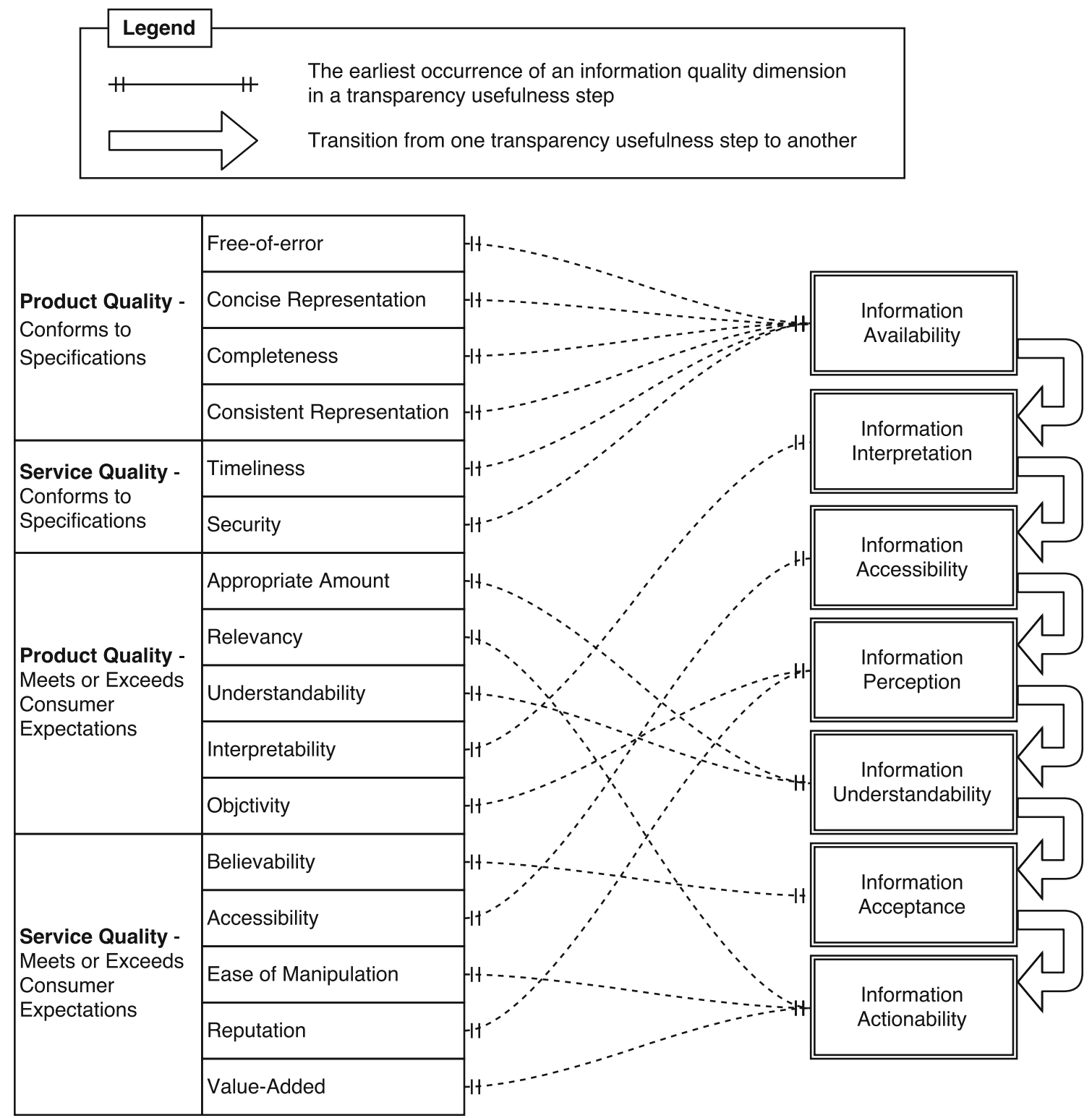

Fig. 9 Interdependencies between quality dimensions and transparency usefulness

necessary technical equipment, or have no access to the internet.

Information receiver is also associated with information perception and information understanding, as they are the recipients of the provided information, and information is perceived and understood by them. Information receiver is also associated with information acceptability and information actionability, as they should decide whether to trust and accept the information, and whether the information can be used in their decision-making or their tasks at hand.

Figure 10 illustrates transparency actors and their potential roles in different steps of transparency usefulness.

\subsection{Interdependencies between transparency usefulness and transparency meaningfulness}

Transparency meaningfulness is treated differently in different steps of achieving useful transparency. In this subsection, we will investigate these interdependencies.

Starting with information availability, it is generally the case that information providers tend to disclose their data more than their processes and policies. There could be several reasons behind this. Some information providers may assume that their recipients simply do not need to know about their processes and policies. In our running example, the public relations office may assume that while 

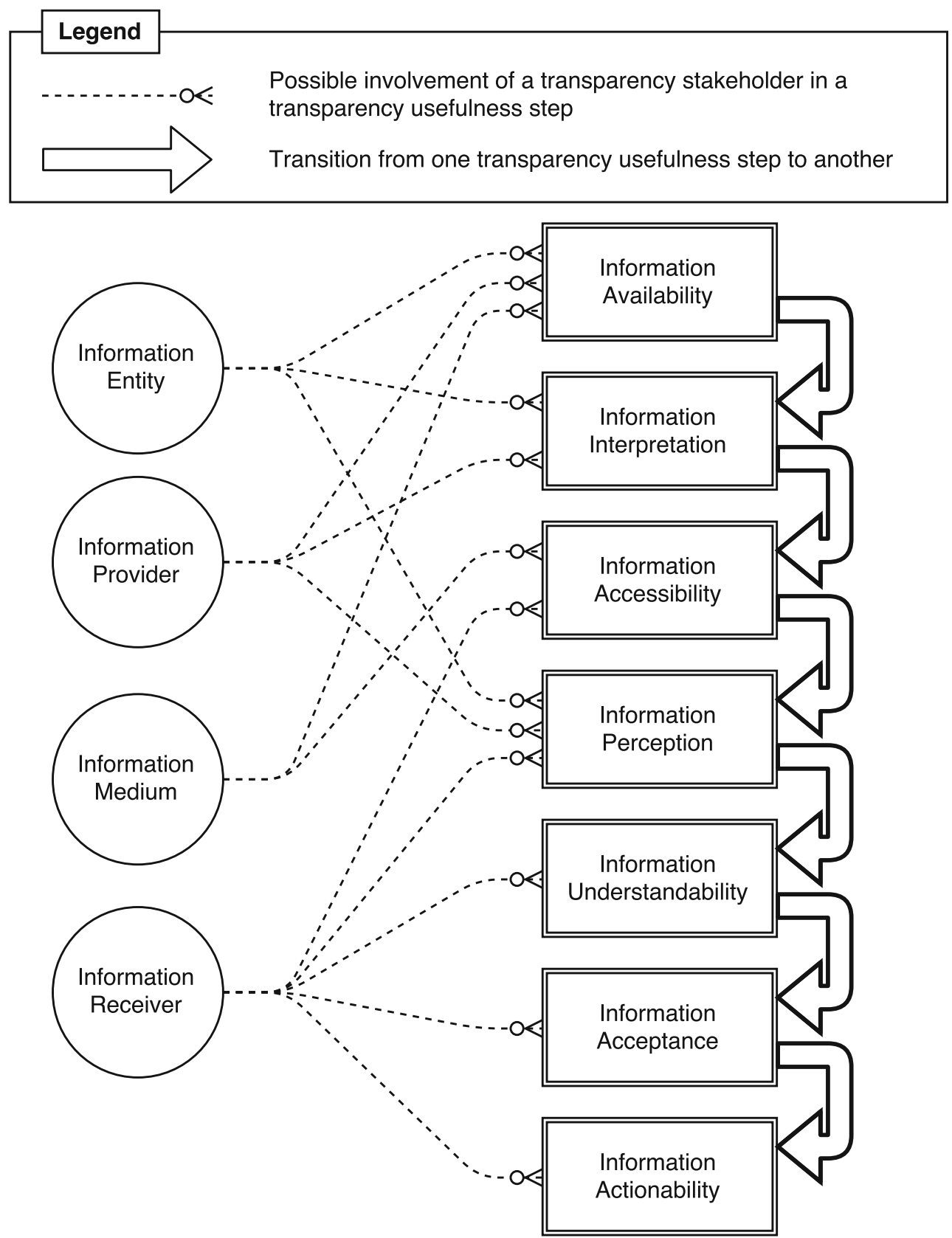

Fig. 10 Interdependencies between transparency usefulness and transparency actors

their customers need to know what financial decisions have been made, they do not need to know how or why they were made. Some information providers might consider such information to be irrelevant to their customers, as they include internal processes. Some may think disclosing such information may cause possible information overload to their customers, leading to more confusion and a decrease in decision-making abilities. Some may even think of such information to be confidential, classified, or unpublishable, as it can decrease their market influence when their competitors also get access to such information.
With regard to information interpretability, information containing data is more interpreted than information containing processes or policies. One reason could be that interpretation is a time-consuming and costly practice [36], and therefore information providers prefer to spend their resources on data interpretation, even though processes and policies may be less straightforward and therefore need more interpretation. In our running example, the public relations office might be more inclined to interpret the data containing the price of shares, their increase or decrease compared to previous years, and future predictions for 
share prices, rather than interpreting how the market dynamics led to an increase or decrease in share prices and why the market dynamics can influence share prices. Furthermore, information containing data is usually more interpretable than information containing processes and policies, as there is simply more data to be presented than processes or policies.

Information accessibility has a direct relationship with information availability, and therefore, data-driven information is generally more accessible than process-driven or policy-driven information.

Information perception is affected by transparency meaningfulness as well, as the data disclosed by information providers is generally more easily perceived than processes or policies disclosed by them. This could be partly due to the fact that processes and policies deal with internal processes that are not necessarily well understood by people [66]. Also, as already stated, data are usually more interpreted than processes and policies, leading to clearer perceptions.

Similar to information perception, data-driven information is better understood by information receivers than process-driven or policy-driven information. The same reasoning for information perception applies to information understandability as well.

With regard to information acceptance, disclosing only data might be less convincing than disclosing processes and policies associated with that data. Knowing the processes and reasons usually makes the information more credible [62] and consequently, more acceptable by information receivers.

Information actionability is also affected by transparency meaningfulness, with the disclosure of processes and policies having a more positive effect and being more influential during decision-making by information receivers.

Figure 11 illustrates how transparency meaningfulness is linked to transparency usefulness in each step.

\section{Case study: UK Freedom of Information Act}

As a proof of concept, in this section we probe the UK Freedom of Information Act 2000 (henceforth FOIA) ${ }^{1}$, which is officially available in the website of the UK government at the time of publication of this article [75]. We investigate FOIA using our four reference models of transparency in order to find the answers to our research questions, while trying to find out if and how it takes into account the information receiver's (i.e., the public in this case) need for transparency. We discuss the lessons learned

\footnotetext{
1 The UK Freedom of Information Act is available at: http://www. legislation.gov.uk.
}

and strengths and weak points found in FOIA in relation to our investigation. Where appropriate, we also propose possible improvements to be considered in newer versions of FOIA.

This case study was conducted as follows. Two principle investigators performed a template analysis on FOIA independently and identified loci where FOIA discussed a concept related to transparency. According to their content, these were then linked to each of the reference models. The initial template used by the investigators included the four reference models and the purpose was to validate, extend, and possibly refine the reference models.

The two investigators then compared results and discussed instances where their outcome was different and settled their arguments. Where the argument could not be settled between the two investigators, a third investigator would join in the discussion in order to conclude the argument. When doubts still remained, a fourth investigator was consulted. When questions were raised related to the meaning of specific terms, the law literature and also the experts in the domain were consulted.

During the template analysis, the investigators aimed to find the answers to two research questions:

1. Does FOIA cover the four reference models? If the answer to this question is positive, to what extent does FOIA cover these four reference models?

2. Does FOIA cover additional aspects of transparency not indicated in the reference models? If the answer to this question is positive, what are those additional aspects of transparency?

During the template analysis, the following constraint was observed. The investigators were looking to answer these research questions within the conceptual modelling remit so that the focus remains on building information systems for managing transparency requirements.

The investigation of FOIA yielded the following answers to the above research questions. It was found out that the answer to the first research question was positive, i.e., FOIA covers some of the aspects of the four reference models. However, the answer to the second research question was negative, i.e., we could not find any transparency facets or properties in FOIA which was not already captured through one of the four reference models. Consequently, in the following subsections, we investigate FOIA with regard to the four reference models and provide a more in-depth answer to the first research question.

\subsection{FOIA and Transparency Actors Wheel}

We investigated FOIA in search of different actors involved in transparency provision. In FOIA, all four actors 


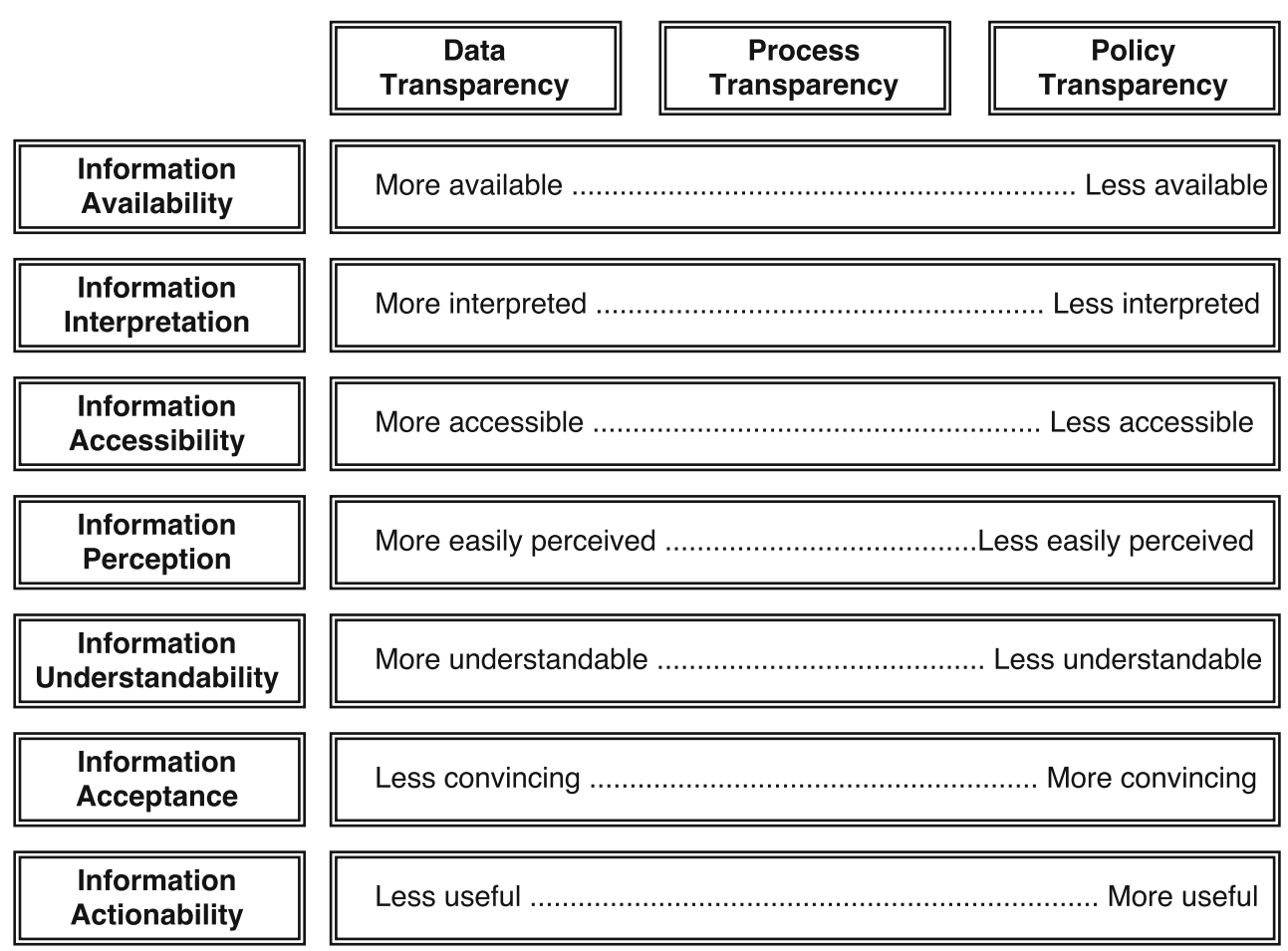

Fig. 11 Interdependencies between transparency usefulness and transparency meaningfulness

identified in Transparency Actors Wheel are present. For example, Part I, Section 1(1), reads:

Any person making a request for information to a public authority is entitled (a) to be informed in writing by the public authority whether it holds information of the description specified in the request, and (b) if that is the case, to have that information communicated to him.

The term "any person" in the above refers to the information receiver and the public authority refers to the information provider. It also mentions that the information should be communicated to the information receiver, therefore acknowledging the presence of an information medium for communication. Information entity is the public office whose information is requested, and an extensive, comprehensive list of them is provided in Schedule 1 of FOIA.

Furthermore, the information which flows amongst different stakeholders is divided into the information which brings about transparency (i.e., transparency-related information) and information held by information provider (i.e., public authority) which does not constitute transparency (i.e., transparency-unrelated information). This can be found in Part I, Section 7(1):

Where a public authority is listed in Schedule 1 only in relation to information of a specified description, nothing in Parts I to $\mathrm{V}$ of this Act applies to any other information held by the authority.

With regard to the transparency classification, FOIA falls into the category of legal demands in demand-side transparency. The reason is that it is the information receiver, and not the information provider, who initiates the transparency provision by demanding for certain information. However, as it is already mentioned, such a legal demand in demand-side transparency produces a mandatory supply-side transparency as well.

\subsection{FOIA and Transparency Depth Pyramid}

In FOIA, it is mainly the data which is communicated to the information receiver. Little mention of processes or policies can be found explicitly in FOIA. In Part I, Section 17(7)(a), FOIA states that:

A notice under subsection (1), (3) or (5) must contain particulars of any procedure provided by the public authority for dealing with complaints about the handling of requests for information or state that the authority does not provide such a procedure.

Furthermore, in Part I, Section 19(3)(b), FOIA states that:

In adopting or reviewing a publication scheme, a public authority shall have regard to the public 
interest in the publication of reasons for decisions made by the authority.

With regard to transparency meaningfulness, the following issues must be considered:

- Even when FOIA does not explicitly mention the communication of processes and policies amongst stakeholders, it is conceivable that the information requested by the information receiver may actually contain them. For example, a Freedom of Information request may concern a city council expenditure on a new bridge which may also contain why the decision on building that bridge was made and how it was made in a council meeting.

- In several occasions in FOIA, it has been duly noted that when the requested information will not be available for information receiver or their choice of information medium is rejected, they should be notified of the reasons for such exemptions from information disclosure or the reasons for refusing the information receiver's communication medium. For example, it is acknowledged in FOIA that it is an obligation of the public authority to notify the information receiver of the reasons for not complying for their preferred method of communication [Part I, Section 11(3)].

\subsection{FOIA and Transparency Achievement Spectrum}

FOIA is mainly concerned with the disclosure of information and information availability. This is justified given the fact that FOIA is meant to deal with the legal requirements of information receivers and is not as much concerned with how such information may or may not help their decision-making processes, and effectively be actionable to them.

Information availability and information accessibility are the two sides of the same coin, representing two different perspectives of information providers and information receivers with regard to the disclosed information. Furthermore, FOIA views information provision as a service (which will be discussed in the next subsection). Therefore, FOIA is also concerned with information accessibility. This can be observed in the title of Part I, which is "Access to Information Held by Public Authorities".

On the other hand, there is no mention in FOIA of information interpretation in a way that can be easily understood by information receivers. Furthermore, FOIA is not concerned with information perception, information understandability, information acceptance, or information actionability. While this is justified, it also means that
FOIA does not necessarily result in useful transparency. Information receivers may receive several hundred pages of data in the form of spreadsheet files and lengthy text files which provide no informational value to them, and in some cases may actually lead to more confusion and possible distrust [55]. For example, in the Freedom of Information section of the website of the UK parliament, ${ }^{2}$ there is a link to transparency publications in which the member of parliaments' expenditures, allowances, and details of finance policies can be found. This obviously satisfies the FOIA regulations, but the provided information is rarely usable for the common audience and needs financial expertise and journalistic endeavours to interpret and understand.

\subsection{FOIA and information quality in transparency}

FOIA is mainly information provider oriented, and as such, there are no mentions of the information quality dimensions that meet or exceed consumer expectations, such as reputation, relevancy, and believability.

FOIA also is product quality agnostic, as it presupposes that the provided information has the standard and expected quality. Therefore, information quality dimensions such as free of error, concise representation, consistent representation, and completeness cannot be found in FOIA either.

We mentioned earlier that FOIA regards information provision as a service. As such, the two information quality dimensions of timeliness and security can be found in FOIA. As for the timeliness of the requested information, Part I, Section 10(1) states:

Subject to subsections (2) and (3), a public authority must comply with section 1(1) promptly and in any event not later than the twentieth working day following the date of receipt.

As for security of the requested information, several loci in Part II of FOIA deal with the exemptions of information provision. Amongst reasons given by FOIA why certain information cannot be disclosed to the public are, to name a few:

- information supplied by, or relating to, bodies dealing with security matters

- information regarding national security

- information regarding the defence of the UK

- information whose disclosure may adversely affect the UK international relations, internal relations within the UK, or the UK economy.

\footnotetext{
2 The Freedom of Information section in the UK parliament website is available at: http://www.parliament.uk/site-information/foi.
} 
On the other hand, FOIA mentions some of the offences related to attempts to the alteration or concealment of information, in Part VIII, Section 77:

... Any person to whom this subsection applies is guilty of an offence if he alters, defaces, blocks, erases, destroys or conceals any record held by the public authority, with the intention of preventing the disclosure by that authority of all, or any part, of the information to the communication of which the applicant would have been entitled.

One can deduce that this article is trying to prevent disinformation or misinformation. However, since several information quality dimensions are not explicitly stated here, or anywhere else in FOIA, it can be concluded that not much attention has been paid to information quality in FOIA other than what was already discussed.

\subsection{Discussion on FOIA}

While investigating FOIA, we also formed several observations with regard to the transparency requirements of stakeholders, the meaningfulness and usefulness of the provided transparency, and other related issues. In this subsection, we share and discuss some of these observations.

\subsubsection{FOIA is mainly associated with mandatory transparency}

FOIA distinctly states that transparency requirements, where legally and pragmatically possible, must be met even when the information is maintained by actors other than the main information provider. In FOIA, Part I, Section 3(2)(a) and 3(2)(b), it is stated that:

For the purpose of this Act, information is held by a public authority if (a) it is held by the authority, otherwise than on behalf of another person, or (b) it is held by another person on behalf of the authority.

This illustrates the importance of meeting transparency requirements as a legal demand of information receivers (i.e., demand-side) and as a legal obligation of information providers (i.e., supply-side).

\subsubsection{It is important to manage occasions where transparency should not be provided}

There are several occasions where transparency requirements cannot be fulfilled, e.g., when the information deals with national security issues and their disclosure might compromise the national security. These occasions are captured and presented in FOIA as instances of information disclosure exemptions. In fact, about 13 pages of FOIA, which constitute Part II of this act, deal with information which is exempt from disclosure, along with several other places in FOIA where the refusal of transparency request is discussed, such as Part I, Section 17. This implies that any model of transparency should also consider loci where transparency provision is prohibited or limited to certain information receivers [53]. This is also in line with our previous research on transparency requirements, where no transparency is considered as the first level of transparency [31], and where constructs have been devised to capture and illustrate the prohibition of information disclosure to certain actors and stakeholders [33].

\subsubsection{Feedback channels should exist between information providers and information receivers}

FOIA acknowledges that in order for information providers to better understand the information required by information receivers, there needs to be a feedback or communication channel. In FOIA, Part I, Section 1(3)(a) and 1(3)(b) state the need for such a feedback channel:

Where a public authority (a) reasonably requires further information in order to identify and locate the information requested, and (b) has informed the applicant of that requirement, the authority is not obliged to comply with subsection (1) unless it is supplied with that further information.

Therefore, stakeholders should be provided with a feedback channel for the efficient management of their transparency requirements. This is in line with our previous research on the importance of utilising structured feedback to elicit stakeholders' transparency requirements [35].

\subsubsection{Managing transparency requirements is costly}

Meeting transparency requirements does not occur without a cost. In fact, meeting transparency requirements can be costly, both in terms of money and in terms of time dedicated to provide transparency. FOIA acknowledges such costs and discusses the possibility of incurring fees on the information receiver's side in Part I, Sections 9 and 13. It also costs time to comply with Freedom of Information requests, which is reflected in Part I, Section 10 of FOIA.

Such costs could potentially discourage both information receivers and information providers from willingly requesting and providing information. Consequently, the automation of the process through software tools and techniques can be a method to reduce both monetary and time costs in the long run. This is in line with our research on the modelling and analysis of transparency requirements and the transparency modelling language we proposed, 
called TranspLan, which benefits from a mathematical notation and automated analysis [33].

\subsubsection{Transparency is meant to be communicated efficiently}

FOIA observes the communication preferences of different information receivers, and obliges information providers to respect such requirements in Part I, Section 11(1)(a):

Where, on making his request for information, the applicant expresses a preference for communication by any one or more of the following means, namely, (a) the provision to the application of a copy of the information in permanent form or in another form acceptable to the applicant ... the public authority shall so far as reasonably practicable give effect to that preference.

With the increasing use of digital devices, it is only reasonable to think that some of these communication channels could include digital devices, such as mobile phones, and digital means, such as email. Consequently, using computerised tools, such as the modelling language proposed for transparency requirements [33], could help increase the efficiency of transparency provision and expand the reach of transparency to more stakeholders.

\subsubsection{Transparency provision can become vexatious}

According to Part I, Section 14 of FOIA, an information receiver cannot make several subsequent identical or substantially similar transparency requests from an information provider (i.e., a public authority). The time and money costs, plus the burden it puts on the shoulders of the information provider, justifies such a prohibition. While this justification is unobjectionable, the automation of the entire procedure of transparency management (e.g., through the use of structured feedback, crowdsourcing, and social adaptation [35]) could remove this obstacle and satisfy information receivers' constant demands for transparency.

\subsubsection{The transparency of transparency requirements can also be problematic}

There are instances where being transparent about why transparency requirements cannot be fulfilled can also be harmful. This is because the disclosure of such information can also reveal information that is classified or should be kept under secrecy regulations, and such disclosure will therefore lead to unwanted transparency. FOIA discusses such a refusal of transparency about transparency in Part I, Section 17(4):
A public authority is not obliged to make a statement under subsection (1)(c) or (3) if, or to the extent that, the statement would involve the disclosure of information which would itself be exempt information.

Therefore, a transparency management tool should also represent these peculiarities of transparency, as is reflected and captured in our transparency modelling language [33].

\section{Conclusion}

In this paper, we presented and discussed four reference models for transparency requirements in information systems. We examined the interdependencies amongst these reference models and how they should be considered during the management of transparency requirements. The reference models were then utilised in order to investigate FOIA and its strengths and weaknesses from an information receiver's perspective, and to recommend amendments where possible. We believe that these reference models together have the potential to capture and manage the peculiarities of transparency requirements, and therefore, they can form a solid foundation for any discourse on transparency requirements.

The reference models on transparency yield several benefits by providing a discourse on transparency requirements which can be used in addressing transparency not only in businesses and their information systems, but also in other domains where information flow occurs. For example, the reference models have been already used to address transparency requirements of people with mild cognitive impairment living in smart homes [2]. The reference models also provide a foundation for systematically investigating transparency requirements from a requirements engineering perspective for elicitation, documentation, and specification. For example, they have proved useful in the design of a transparency modelling language, called TranspLan, which aims to model and analyse the transparency requirements of stakeholders in a business information system [33].

There are several possible future works based on these reference models. One possibility is to validate and evaluate the reference models, e.g., by using an ontological approach [19]. Another possible future work is to apply the reference models in other domains where the transparency of information is relevant and important, similar to the work already conducted on the transparency requirements of people with mild cognitive impairment in ambient intelligent environments. Researchers from various fields of study, such as management and law, may also use these reference models and their terminology to enrich their communication on transparency, because these reference 
models provide them with a rich vocabulary with shared understanding.

Acknowledgements The research is supported by an FP7 Marie Curie CIG grant (the SOCIAD project) and Bournemouth University through the Fusion Investment Fund.

Open Access This article is distributed under the terms of the Creative Commons Attribution 4.0 International License (http://crea tivecommons.org/licenses/by/4.0/), which permits unrestricted use, distribution, and reproduction in any medium, provided you give appropriate credit to the original author(s) and the source, provide a link to the Creative Commons license, and indicate if changes were made.

\section{References}

1. Ali R, Solis C, Omoronyia I, Salehie M, Nuseibeh B (2012) Social adaptation: when software gives users a voice. In: Proceedings of the seventh international conference on evaluation of novel approaches to software engineering (ENASE)

2. Amiribesheli M, Hosseini M, Bouchachia A (2016) A user-centred principle based transparency approach for intelligent environments. In: Proceedings of the human centred design for intelligent environments workshop at British $\mathrm{HCI}$ conference

3. Araujo R, Taher Y, Heuvel W, Cappelli C (2013) Evolving government-citizen ties in public service design and delivery. In: Proceedings of the twelfth international federation of information processing e-government conference, pp 19-26

4. Bannister F, Connolly R (2011) The trouble with transparency: a critical review of openness in e-government. Policy Internet 3(1):1-30

5. Bellver A, Kaufmann D (2005) Transparenting transparency: initial empirics and policy applications. World bank policy research working paper, pp 1-72

6. Bok S (1989) Secrets: on the ethics of concealment and revelation. Vintage, New York, United States

7. Cappelli C, Cunha H, Gonzalez-Baixauli B, do Prado Leite JCS (2010) Transparency versus security: early analysis of antagonistic requirements. In: Proceedings of the 2010 ACM symposium on applied computing, pp 298-305

8. Cappelli C, Leite do Prado Leite JCS, Oliveira ADPA (2007) Exploring business process transparency concepts. In: Proceedings of the 15th IEEE international requirements engineering conference (RE), pp 389-390

9. Chung L, Nixon BA, Yu E, Mylopoulos J (2012) Non-functional requirements in software engineering, vol 5. Springer Science \& Business Media, Berlin, Germany

10. Cole M, Gay J, Glick J, Sharp D (1971) The cultural context of learning and thinking: an exploration in experimental anthropology. Basic Books Inc, New York, United States

11. Greeno JG, Collins AM, Resnick LB (1996) Cognition and learning. Handb Educ Psychol 77:15-46

12. Curtin D, Meijer A (2006) Does transparency strengthen legitimacy? A critical analysis of european union policy documents. Inf Polity 11:109-122

13. Dabbish L, Stuart C, Tsay J, Herbsleb J (2012) Social coding in github: transparency and collaboration in an open software repository. In: Proceedings of computer supported cooperative work (CSCW) conference, pp 1277-1286

14. Dabbish L, Stuart C, Tsay J, Herbsleb J (2013) Leveraging transparency. IEEE Softw 30(1):37-43
15. Dawes SS (2010) Stewardship and usefulness: policy principles for information-based transparency. Gov Inf Q 27(4):377-383

16. do Prado Leite JCS, Cappelli C (2008) Exploring $i^{*}$ characteristics that support software transparency. In: Proceedings of the third international $i *$ workshop, pp 51-54

17. do Prado Leite JCS, Cappelli C (2010) Software transparency. Bus Inf Syst Eng 2(3):127-139

18. Etzioni A (2010) Is transparency the best disinfectant? J Polit Philos 18(4):389-404

19. Fettke P, Loos P (2003) Ontological evaluation of reference models using the bunge-wand-weber model. In: Proceedings of the ninth Americas conference on information systems, p 384

20. Florini A, Birdsall N, Flynn S, Haufler V, Lipton D, Morrow D, Sharma S (2000) Does the invisible hand need a transparent glove? The politics of transparency. In: Proceedings of the world banks annual conference on development economics, pp 163-184

21. Fox J (2007) The uncertain relationship between transparency and accountability. Dev Pract 17(4-5):663-671

22. Frentrup M, Theuvsen L (2006) Transparency in supply chains: is trust a limiting factor? In: Proceedings of the 99th European association of agricultural economists (EAAE) seminar trust and risk in business networks', pp 65-74

23. Fuller MA, Serva MA, Benamati J et al (2007) Seeing is believing: the transitory influence of reputation information on e-commerce trust and decision making. Decis Sci 38(4):675-699

24. Ghanavati S, Amyot D, Peyton L (2007) Towards a framework for tracking legal compliance in healthcare. In: Proceedings of the 19th international conference on advanced information systems engineering (CAiSE). Springer, pp 218-232

25. Gower KK (2006) Truth and transparency. In: Ethics in public relations. SAGE Publicaions, United States, pp 89-105

26. Griffith JC (2006) Beyond transparency: new standards for legislative information systems. European Centre for Parliamentary Research and Documentation, Geneva, Switzerland

27. Grimmelikhuijsen S (2012) Linking transparency, knowledge and citizen trust in government: an experiment. Int Rev Adm Sci 78(1):50-73

28. Harrison TM, Guerrero S, Burke GB, Cook M, Cresswell A, Helbig N, Hrdinová J, Pardo T (2011) Open government and e-government: democratic challenges from a public value perspective. In: Proceedings of the 12th international digital government research conference, pp 245-253

29. Hedbom H (2008) A survey on transparency tools for enhancing privacy. In: IFIP summer school on the future of identity in the information society. Springer, Berlin, pp 67-82

30. Holzner B, Holzner L (2006) Transparency in global change: the vanguard of the open society. University of Pittsburgh Press, Pittsburgh

31. Hosseini M, Shahri A, Phalp K, Ali R (2015) Towards engineering transparency as a requirement in socio-technical systems. In: Proceedings of the 23rd IEEE international requirements engineering conference (RE)

32. Hosseini M, Shahri A, Phalp K, Ali R (2016) Foundations for transparency requirements engineering. In: Proceedings of the 22nd international working conference on requirements engineering: foundation for software quality (REFSQ). Springer, pp 225-231

33. Hosseini M, Shahri A, Phalp K, Ali R (2016) A modelling language for transparency requirements in business information systems. In: Proceedings of the 28th international conference on advanced information systems engineering (CAiSE). Springer, pp. 239-254

34. Hosseini M, Shahri A, Phalp KT, Ali R (2015) Transparency as a requirement. In: Joint proceedings of REFSQ 2015 workshops, research method track, and poster track, co-located with the 21 st 
international conference on requirements engineering: foundation for software quality (REFSQ)

35. Hosseini M, Shahri A, Phalp KT, Ali R (2016) Crowdsourcing transparency requirements through structured feedback and social adaptation. In: Proceedings of the 10th IEEE international conference on research challenges in information science (RCIS)

36. Indjejikian RJ (1991) The impact of costly information interpretation on firm disclosure decisions. J Account Res 29(2):277-301. doi:10.2307/2491050

37. Ingolfo S, Siena A, Mylopoulos J, Susi A, Perini A (2013) Arguing regulatory compliance of software requirements. Data Knowl Eng 87:279-296

38. Jessup LM, Valacich JS (2002) Information systems today. Prentice hall professional technical reference

39. Kachalia A (2013) Improving patient safety through transparency. N Engl J Med 369(18):1677-1679

40. Kahn BK, Strong DM, Wang RY (2002) Information quality benchmarks: product and service performance. Commun ACM 45(4):184-192

41. Kearney AR (1994) Understanding global change: a cognitive perspective on communicating through stories. Clim Change 27(4):419-441

42. Kolstad I, Wiig A (2009) Is transparency the key to reducing corruption in resource-rich countries? World Dev 37(3):521-532

43. Kroenke D (2011) MIS essentials. Prentice Hall Press, Upper Saddle River

44. Lindstedt C, Naurin D (2010) Transparency is not enough: making transparency effective in reducing corruption. Int Polit Sci Rev 31(3):301-322

45. Lodge M (2004) Accountability and transparency in regulation: critiques, doctrines and instruments. Edward Elgar Publishing, Cheltenham

46. MacKenzie CM, Laskey K, McCabe F, Brown PF, Metz R, Hamilton BA (2006) Reference model for service oriented architecture 1.0. OASIS standard 12

47. McManus T, Holtzman Y, Lazarus H, Anderberg J, Simon C (2006) Corporate information transparency: the synthesis of internal and external information streams. J Manag Dev 25(10):1029-1031

48. Meijer A (2009) Understanding modern transparency. Int Rev Adm Sci 75(2):255-269

49. Meunier P (2008) Software transparency and purity. Commun ACM 51(2):104-104

50. Michener G, Bersch K (2011) Conceptualizing the quality of transparency. In: Proceedings of the first global conference on transparency

51. Mitchell R (1998) Sources of transparency: information systems in international regimes. Int Stud Q 42(1):109-130. http://www. jstor.org/stable/2600819

52. Moghaddam S, Jamali M, Ester M, Habibi J (2009) Feedbacktrust: using feedback effects in trust-based recommendation systems. In: Proceedings of the third ACM conference on recommender systems, pp 269-272

53. O'Hara K (2011) Transparent government, not transparent citizens: a report on privacy and transparency for the cabinet office. Commissioned by the Cabinet Office, United Kingdom

54. Ojameruaye B, Bahsoon R (2014) Systematic elaboration of compliance requirements using compliance debt and portfolio theory. In: Proceedings of the 20th international working conference requirements engineering: foundation for software quality (REFSQ). Springer, pp 152-167

55. O'Reilly CA (1980) Individuals and information overload in organizations: is more necessarily better? Acad Manag J 23(4):684-696

56. Osborne D (2004) Transparency and accountability reconsidered. J Financ Crime 11(3):292-300
57. Pronin E, Gilovich T, Ross L (2004) Objectivity in the eye of the beholder: divergent perceptions of bias in self versus others. Psychol Rev 111(3):781

58. Rawlins B (2008) Give the emperor a mirror: toward developing a stakeholder measurement of organizational transparency. J Public Relat Res 21(1):71-99

59. Ruppert T, Bernard J, Kohlhammer J (2013) Bridging knowledge gaps in policy analysis with information visualization. Electronic government and electronic participation: joint proceedings of ongoing research of international federation for information processing e-government (IFIP EGOV) and international federation for information processing ePart 221, pp 92-103

60. Scauer F (2011) Transparency in three dimensions. University of Illinois Law Review, United States, p 1339

61. Schnackenberg AK, Tomlinson EC (2014) Organizational transparency a new perspective on managing trust in organizationstakeholder relationships. J Manag 1784-1810

62. Scott T (1994) Incentives and disincentives for financial disclosure: voluntary disclosure of defined benefit pension plan information by canadian firms. Account Rev 69(1):26-43. http://www. jstor.org/stable/248259

63. Serrano M, do Prado Leite JCS (2011) Capturing transparencyrelated requirements patterns through argumentation. In: Proceedings of the first international workshop on requirements patterns $(\mathrm{RePa})$, pp 32-41

64. Shkabatur J (2012) Transparency with (out) accountability: open government in the united states. Yale Law Policy Rev 31(1):79-140

65. Sinha R, Swearingen K (2002) The role of transparency in recommender systems. In: Extended abstracts of the 2002 conference on human factors in computing systems (CHI), pp 830-831

66. Stauss B (2000) Using new media for customer interaction: a challenge for relationship marketing. In: Hennig-Thurau $\mathrm{T}$, Hansen U (eds) Relationship marketing. Springer, Berlin, pp 233-253

67. Stirton L, Lodge M (2001) Transparency mechanisms: building publicness into public services. J Law Soc 28(4):471-489

68. Streufert SC (1973) Effects of information relevance on decision making in complex environments. Mem Cogn 1(3):224-228

69. Stuart HC, Dabbish L, Kiesler S, Kinnaird P, Kang R (2012) Social transparency in networked information exchange: a theoretical framework. In: Proceedings of the ACM computer supported cooperative work (CSCW) conference, pp 451-460

70. Svenson O (1979) Process descriptions of decision making. Organ Behav Hum Perform 23(1):86-112

71. Swann WB, Hill CA (1982) When our identities are mistaken: reaffirming self-conceptions through social interaction. J Personal Soc Psychol 43(1):59

72. Tagiuri R, Kogan N, Bruner J (1955) The transparency of interpersonal choice. Sociometry 18(4):368-379. doi:10.2307/2785873

73. Tanimoto S (2005) Dimensions of transparency in open learner models. In: Proceedings of internatioal workshop on learner modelling for reflection

74. Tidline TJ (1999) The mythology of information overload. Libr Trends 47(3):485-506

75. United Kingdom Government: Freedom of Information Act 2000. [Online] (2000). http://www.legislation.gov.uk/ukpga/2000/36/ contents. Last Accessed 20 Dec 202016

76. Vaccaro A, Madsen P (2006) Firm information transparency: ethical questions in the information age. In: International federation for information processing (IFIP) international conference on human choice and computers. Springer, pp 145-156

77. Vaccaro A, Madsen P (2009) Corporate dynamic transparency: the new ict-driven ethics? Ethics Inf Technol 11(2):113-122

78. Van der Cruijsen CA, Eijffinger SC (2010) From actual to perceived transparency: the case of the european central bank. J Econ Psychol 31(3):388-399 
79. Venkatesh V, Morris M, Davis G, Davis F (2003) User acceptance of information technology: toward a unified view. MIS Q 27(3):425-478. http://www.jstor.org/stable/30036540

80. Wall SP (1996) Public justification and the transparency argument. Philos Q (1950) 46(185):501-507. doi:10.2307/2956360

81. Williams C (2000) Text of remarks on panel: codes of conduct and transparency. Hastings Int Comp Law Rev 24:415
82. Wolfe R (2003) Regulatory transparency, developing countries and the wto. World Trade Rev 2(02):157-182

83. Yu E (1994) Modelling strategic relationships for process reengineering. Ph.D. thesis, Graduate Department of Computer Science, University of Toronto 